\title{
Cut Finite Element Methods for Coupled Bulk-Surface Problems
}

\author{
Erik Burman ${ }^{* \dagger}$ Peter Hansbo $\ddagger \S$ Mats G. Larson $9 \|$ Sara Zahedi **
}

\begin{abstract}
We develop a cut finite element method for a second order elliptic coupled bulksurface model problem. We prove a priori estimates for the energy and $L^{2}$ norms of the error. Using stabilization terms we show that the resulting algebraic system of equations has a similar condition number as a standard fitted finite element method. Finally, we present a numerical example illustrating the accuracy and the robustness of our approach.
\end{abstract}

\section{Introduction}

Problems involving phenomena that take place both on surfaces (or interfaces) and in bulk domains occur in a variety of applications in fluid dynamics and biological applications. An example is given by the modeling of soluble surfactants. Surfactants are important because of their ability to reduce the surface tension. Examples of applications where the effects of surfactants are important in the modelling include detergents, oil recovery, and the treatment of lung diseases. A soluble surfactant is dissolved in the bulk fluid but also exists in adsorbed form on the interface. A computational challenge is then to properly account for the exchange between these two surfactant forms. The coupling between the dissolved form in the bulk and the adsorbed form on the interface involves computations of the gradient of the bulk surfactant concentration on a moving interface that may undergo topological changes, see e.g. [1]. In this context computational methods that allow the interface to be arbitrarily located with respect to a fixed background mesh are of great interest.

*Department of Mathematics, University College London, London, UK-WC1E 6BT, United Kingdom

${ }^{\dagger}$ Supported by EPSRC, UK, Grant Nr. EP/J002313/1.

${ }^{\ddagger}$ Department of Mechanical Engineering, Jönköping University, SE-55111 Jönköping, Sweden.

$\S$ Supported by the Swedish Foundation for Strategic Research Grant Nr. AM13-0029 and the Swedish Research Council Grant Nr. 2011-4992.

IDepartment of Mathematics and Mathematical Statistics, Umeå University, SE-90187 Umeå, Sweden

"Supported by the Swedish Foundation for Strategic Research Grant Nr. AM13-0029 and the Swedish Research Council Grant Nr. 2013-4708.

** Department of Mathematics, KTH Royal Institute of Technology, SE-100 44 Stockholm, Sweden 
We consider a basic model problem of this nature that involves two coupled elliptic problems one in the bulk and one on the boundary of the bulk domain. The coupling term is defined in such a way that the overall bilinear form in the corresponding weak statement is coercive. A finite element method was proposed and analyzed for a similar model problem in [7]. See also [6], and the references therein for background on finite element methods for partial differential equations on surfaces. In [7] a polyhedral approximation of the bulk domain was used and its piecewise polynomial boundary faces served as approximation of the surface. In this contribution we develop a method that is unfitted, that is, the surface is allowed to cut through a fixed background mesh in an arbitrary way. Such a finite element method was proposed in [12] for the Laplace-Beltrami operator. A general framework for this type of computational methods using finite element methods on cut meshes, co called CutFEM methods was recently discussed in [3]. The CutFEM approach is convenient since the same finite element space defined on a background grid can be used for solving both the partial differential equation in the bulk region and on the surface. However, a drawback of this type of methods is that the stiffness matrix may become arbitrarily ill conditioned depending on the position of the surface in the background mesh. In the case of the Laplace-Beltrami operator this ill conditioning has been addressed in [13] and [5]. For results on the stability of the bulk equation on cut meshes see [4, 10, 11].

We use continuous piecewise linear elements defined on the background mesh to solve both the problem in the bulk domain and the problem on the surface. To stabilize the method we add gradient jump penalty terms as in [4, 5] that ensure that the resulting algebraic system of equations has optimal condition number. We also consider the approximation of the domain and prove a priori error estimates in both the $H^{1}$ - and $L^{2}{ }^{-}$norms, taking both the approximation of the domain and of the solution into account.

The remainder of the paper is outlined as follows: In Section 2 we introduce the model problem and state the weak form, in Section 3 we introduce a discrete approximation of the domain, in Section 4 we prove a priori estimates for the energy and $L^{2}$ norm of the error, in Section 5 we prove an estimate of the condition number, and finally in Section 6 we present a numerical example.

\section{The Continuous Coupled Bulk-Surface Problem}

\subsection{Strong Form}

Let $\Omega$ be a domain in $\mathbb{R}^{3}$ with smooth boundary $\Gamma$ and exterior unit normal $n$. We consider the following problem: find $u_{B}: \Omega \rightarrow \mathbb{R}$ and $u_{S}: \Gamma \rightarrow \mathbb{R}$ such that

$$
\begin{aligned}
-\nabla \cdot\left(k_{B} \nabla u_{B}\right) & =f_{B} & & \text { in } \Omega \\
-n \cdot k_{B} \nabla u_{B} & =b_{B} u_{B}-b_{S} u_{S} & & \text { on } \Gamma \\
-\nabla_{\Gamma} \cdot\left(k_{S} \nabla_{\Gamma} u_{S}\right) & =f_{S}-n \cdot k_{B} \nabla u_{B} & & \text { on } \Gamma
\end{aligned}
$$

Here $\nabla$ is the $\mathbb{R}^{3}$ gradient and $\nabla_{\Gamma}$ is the tangent gradient associated with $\Gamma$ defined by

$$
\nabla_{\Gamma}=P_{\Gamma} \nabla
$$


with $P_{\Gamma}=P_{\Gamma}(x)$ the projection of $\mathbb{R}^{3}$ onto the tangent plane of $\Gamma$ at $x \in \Gamma$, defined by

$$
P_{\Gamma}=I-n \otimes n
$$

Further, $b_{B}, b_{S}, k_{B}$, and $k_{S}$ are positive constants, and $f_{B}: \Omega \rightarrow \mathbb{R}$ and $f_{S}: \Gamma \rightarrow \mathbb{R}$ are given functions. As mentioned above, this problem serves as a basic model for the concentration of surfactants interacting with a bulk concentration; it also models other processes, e.g., proton transport via a membrane surface [9].

\subsection{Weak Form}

Multiplying (2.1) by $v_{B} \in H^{1}(\Omega)$, integrating by parts, and using the boundary condition (2.2), we obtain

$$
\begin{aligned}
\left(f_{B}, v_{B}\right)_{\Omega} & =\left(k_{B} \nabla u_{B}, \nabla v_{B}\right)_{\Omega}-\left(n \cdot k_{B} \nabla u_{B}, v_{B}\right)_{\Gamma} \\
& =\left(k_{B} \nabla u_{B}, \nabla v_{B}\right)_{\Omega}+\left(b_{B} u_{B}-b_{S} u_{S}, v_{B}\right)_{\Gamma}
\end{aligned}
$$

and thus we have the weak statement

$$
\left(k_{B} \nabla u_{B}, \nabla v_{B}\right)_{\Omega}+\left(b_{B} u_{B}-b_{S} u_{S}, v_{B}\right)_{\Gamma}=\left(f_{B}, v_{B}\right)_{\Omega} \quad \forall v_{B} \in H^{1}(\Omega)
$$

Next multiplying (2.3) by $v_{S} \in H^{1}(\Gamma)$, integrating by parts, and again using (2.2) we obtain

$$
\begin{aligned}
\left(k_{S} \nabla_{\Gamma} u_{S}, \nabla_{\Gamma} v_{S}\right)_{\Gamma} & =\left(f_{S}-n \cdot k_{S} \nabla u_{B}, v_{S}\right)_{\Gamma} \\
& =\left(f_{S}+\left(b_{B} u_{B}-b_{S} u_{S}\right), v_{S}\right)_{\Gamma}
\end{aligned}
$$

and thus

$$
\left(k_{S} \nabla u_{S}, \nabla v_{S}\right)_{\Gamma}-\left(b_{B} u_{B}-b_{S} u_{S}, v_{S}\right)_{\Gamma}=\left(f_{S}, v_{S}\right)_{\Gamma} \quad \forall v_{S} \in H^{1}(\Gamma)
$$

We note that the solution to this system of equations is uniquely determined up to a pair of constant functions $\left(c_{B}, c_{S}\right)$ such that $b_{B} c_{B}-b_{S} c_{S}=0$. To obtain a unique solution we here choose to enforce $\int_{\Gamma} u_{S}=0$.

Introducing the function spaces

$$
V_{B}=H^{1}(\Omega), \quad V_{S}=H^{1}(\Gamma) /\left\langle 1_{\Gamma}\right\rangle, \quad W=V_{B} \times V_{S}
$$

and choosing the test functions $b_{B} v_{B}$ and $b_{S} v_{S}$ we get the variational problem: find $u=$ $\left(u_{B}, u_{S}\right) \in W$ such that

$$
a(u, v)=l(v) \quad \forall v \in W
$$

Here

$$
a(u, v)=a_{B}\left(u_{B}, v_{B}\right)+a_{S}\left(u_{S}, v_{S}\right)+a_{B S}(u, v)
$$


with

$$
\left\{\begin{array}{l}
a_{B}\left(u_{B}, v_{B}\right)=b_{B}\left(k_{B} \nabla u_{B}, \nabla v_{B}\right)_{\Omega} \\
a_{S}\left(u_{S}, v_{S}\right)=b_{S}\left(k_{S} \nabla_{S} u_{S}, \nabla v_{S}\right)_{\Gamma} \\
a_{B S}(u, v)=\left(b_{B} u_{B}-b_{S} u_{S}, b_{B} v_{B}-b_{S} v_{S}\right)_{\Gamma}=(b \cdot u, b \cdot v)_{\Gamma}
\end{array}\right.
$$

where we also introduced the notation $b=\left(b_{B},-b_{S}\right)$ and

$$
l(v)=l_{B}\left(v_{B}\right)+l_{S}\left(v_{S}\right)=b_{B}\left(f_{B}, v_{B}\right)_{\Omega}+b_{S}\left(f_{S}, v_{S}\right)_{\Gamma}
$$

Introducing the energy norm

$$
\|u\|^{2}=a(u, u)
$$

we directly obtain coercivity and continuity of the bilinear form $a(\cdot, \cdot)$ and continuity of $l(\cdot)$. Using Lax-Milgram there is a unique solution in $W$. If $\Gamma$ is $C^{3}$ we additionally have the elliptic regularity estimate

$$
\left\|u_{B}\right\|_{H^{2}(\Omega)}+\left\|u_{S}\right\|_{H^{2}(\Gamma)} \lesssim\left\|f_{B}\right\|_{L^{2}(\Omega)}+\left\|f_{S}\right\|_{L^{2}(\Gamma)}
$$

see [7] for details. Here and below $\lesssim$ denotes less or equal up to a constant, $\|\cdot\|_{H^{s}(\omega)}$ denotes the standard Sobolev norm $H^{s}(\omega)$ norm on the set $\omega$, and $\|\cdot\|_{L^{p}(\omega)}$ denotes the $L^{p}(\omega)$ norm.

\section{The Finite Element Method}

\subsection{Approximation of the Domain}

Let $p: \mathbb{R}^{3} \ni x \mapsto \operatorname{argmin}_{y \in \Gamma}|y-x| \in \Gamma$ denote the closest point mapping. Then there is an open neighborhood $\mathcal{U}(\Gamma)$ of $\Gamma$ such that for each $x \in \mathcal{U}(\Gamma)$ there is a uniquely determined $p(x) \in \Gamma$. We let $\rho$ be the signed distance function, $\rho(x)=|p(x)-x|$ in $\mathbb{R}^{3} \backslash \Omega$ and $\rho(x)=-|p(x)-x|$ in $\Omega$. We define the extension of any function define on $\Gamma$ to $\mathcal{U}(\Gamma)$ as follows

$$
v^{e}=v \circ p
$$

Let $\Omega_{0}$ be a domain in $\mathbb{R}^{3}$ that contains $\Omega \cup \mathcal{U}(\Gamma)$ and let $\mathcal{K}_{0, h}$ be a quasiuniform partition of $\Omega_{0}$ into shape regular tetrahedra with mesh parameter $h$. See Fig. 1 for an illustration of the different domains. We consider a continuous piecewise linear approximation $\Gamma_{h}$ of $\Gamma$ such that $\Gamma_{h} \cap K$ is a subset of a hyperplane in $\mathbb{R}^{3}$ for each $K \in \mathcal{K}_{0, h}$.

We assume that $\Gamma_{h} \subset \mathcal{U}(\Gamma)$ and that the following approximation assumptions hold:

$$
\|\rho\|_{L^{\infty}\left(\Gamma_{h}\right)} \lesssim h^{2}
$$

and

$$
\left\|n^{e}-n_{h}\right\|_{L^{\infty}\left(\Gamma_{h}\right)} \lesssim h
$$

where $n_{h}$ denotes the piecewise constant exterior unit normal to $\Gamma_{h}$. Finally, we define $\Omega_{h}$ as the domain enclosed by $\Gamma_{h}$. These assumptions are consistent with the piecewise linear nature of the discrete surface. 


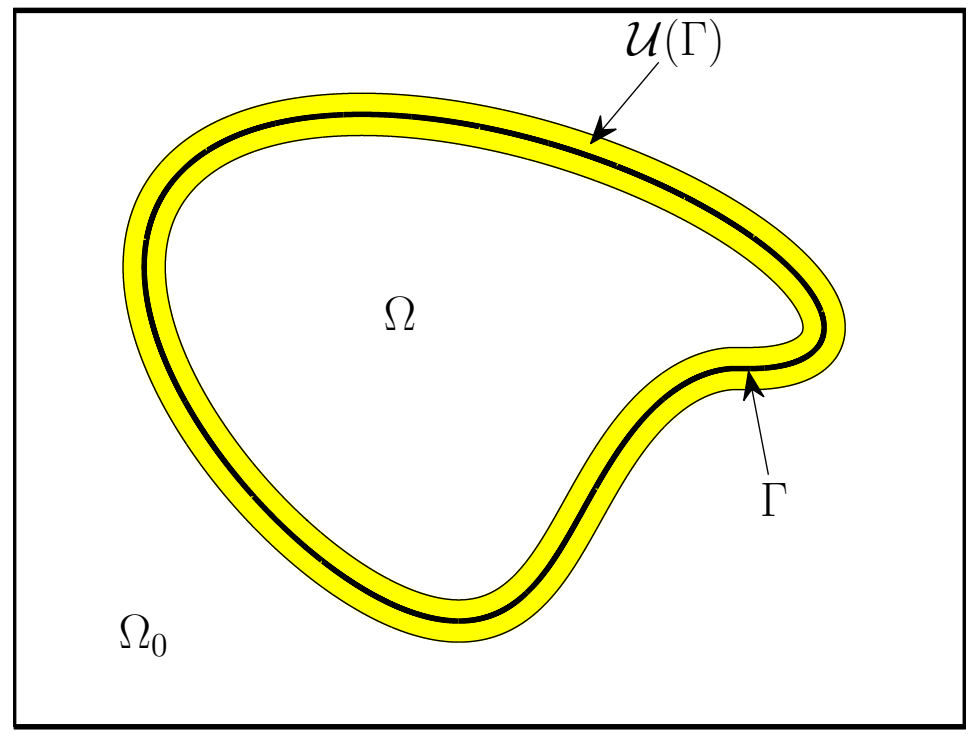

Figure 1: Illustration of the domain $\Omega, \Omega_{0}, \mathcal{U}(\Gamma)$, and $\Gamma$. The domain $\mathcal{U}(\Gamma)$ is the yellow region where for each $x \in \mathcal{U}(\Gamma)$ there is a unique closest point on $\Gamma$.

\subsection{Finite Element Spaces}

We define the following sets of elements

$$
\mathcal{K}_{B, h}=\left\{K \in \mathcal{K}_{h, 0}: K \cap \Omega_{h} \neq \emptyset\right\}, \quad \mathcal{K}_{S, h}=\left\{K \in \mathcal{K}_{h, 0}: K \cap \Gamma_{h} \neq \emptyset\right\}
$$

and the corresponding sets

$$
\mathcal{N}_{B, h}=\bigcup_{K \in \mathcal{K}_{B, h}} K, \quad \mathcal{N}_{S, h}=\bigcup_{K \in \mathcal{K}_{S, h}} K
$$

We let $V_{0, h}$ be the space of piecewise linear continuous functions defined on $\mathcal{K}_{0, h}$. Next let

$$
V_{B, h}=\left.V_{0, h}\right|_{\mathcal{N}_{B, h}}, \quad V_{S, h}=\left.V_{0, h}\right|_{\mathcal{N}_{S, h}} /\left\langle 1_{\Gamma_{h}}\right\rangle, \quad W_{h}=V_{B, h} \times V_{S, h}
$$

be the spaces of continuous piecewise linear polynomials defined on $\mathcal{N}_{B, h}$ and $\mathcal{N}_{S, h}$, respectively, where we also enforced $\int_{\Gamma_{h}} v_{S}=0$ for $v \in V_{S, h}$.

\subsection{The Finite Element Method}

The finite element method takes the form: find $u_{h}=\left(u_{B, h}, u_{S, h}\right) \in W_{h}$ such that

$$
A_{h}\left(u_{h}, v\right)=l_{h}(v) \quad \forall v \in W_{h}
$$


Here the bilinear form is defined by

$$
A_{h}(v, w)=a_{h}(v, w)+j_{h}(v, w)
$$

with

$$
a_{h}(v, w)=a_{B, h}\left(v_{B}, w_{B}\right)+a_{S, h}\left(v_{S}, w_{S}\right)+a_{B S, h}(v, w)
$$

and

$$
\left\{\begin{array}{l}
a_{B, h}\left(u_{B}, v_{B}\right)=b_{B}\left(k_{B} \nabla u_{B}, \nabla v_{B}\right)_{\Omega_{h}} \\
a_{S, h}\left(u_{S}, v_{S}\right)=b_{S}\left(k_{S} \nabla_{S} u_{S}, \nabla_{S} v_{S}\right)_{\Gamma_{h}} \\
a_{B S, h}(u, v)=\left(b_{B} u_{B}-b_{S} u_{S}, b_{B} v_{B}-b_{S} v_{S}\right)_{\Gamma_{h}}=(b \cdot u, b \cdot v)_{\Gamma_{h}}
\end{array}\right.
$$

where $\nabla_{\Gamma_{h}}=P_{h} \nabla$ and $P_{h}=I-n_{h} \otimes n_{h}$. Next $j_{h}(v, w)$ is a stabilizing term of the form

$$
j_{h}(v, w)=\tau_{B} h^{3} j_{B}\left(v_{B}, w_{B}\right)+\tau_{S} j_{S}\left(v_{S}, w_{S}\right)
$$

where $\tau_{B}, \tau_{S}$ are positive parameters and, letting $\left.[x]\right|_{F}$ denote the jump of $x$ over the face $F$,

$$
\begin{aligned}
j_{B}\left(v_{B}, w_{B}\right) & =\sum_{F \in \mathcal{F}_{B, h}}\left(\left[n_{F} \cdot \nabla v_{B}\right],\left[n_{F} \cdot \nabla w_{B}\right]\right)_{F} \\
j_{S}\left(v_{S}, w_{S}\right) & =\sum_{F \in \mathcal{F}_{S, h}}\left(\left[n_{F} \cdot \nabla v_{S}\right],\left[n_{F} \cdot \nabla w_{S}\right]\right)_{F}
\end{aligned}
$$

with $\mathcal{F}_{S, h}$ the set of internal faces (i.e. faces with two neighbors) in $\mathcal{K}_{S, h}$ and $\mathcal{F}_{B, h}$ denotes the set of faces that are internal in $\mathcal{K}_{B, h}$ and belong to an element in $\mathcal{K}_{S, h}$. Finally, the right hand side is defined by

$$
l_{h}(v)=l_{B, h}\left(v_{B}\right)+l_{S, h}\left(v_{S}\right)=b_{B}\left(f_{B, h}, v_{B}\right)_{\Omega_{h}}+b_{S}\left(f_{S, h}, v_{S}\right)_{\Gamma_{h}}
$$

with $f_{B, h}$ and $f_{S, h}$ discrete approximations of $f_{B}$ and $f_{S}$ that will be specified more precisely below.

The purpose of the stabilization terms is to ensure that the resulting algebraic system of equations is well conditioned.

\section{A Priori Error Estimates}

Outline of the proof. To prove a priori error estimates we first construct a bijective mapping $F_{h}$ that maps the exact domain to the approximate domain. The mapping is used to lift the discrete solution onto the exact domain where the error is evaluated. The construction of the mapping is based on a representation of the discrete boundary $\Gamma_{h}$ as a normal function over the exact boundary $\Gamma$ together with an extension to a small tubular $\delta$ neighborhood of the boundary. In the remainder of the domain $F_{h}$ is the identity mapping. Next a Strang type lemma relates the error in the computed solution to an interpolation 
error and quadrature errors emanating from the approximation of the domain. Using the assumptions on the approximation properties of the discrete surface we derive bounds on the quadrature errors. The surface quadrature errors are $O\left(h^{2}\right)$ while the bulk quadrature error is $O(h)$ in the $\delta$ neighborhood and zero elsewhere. To establish an optimal order energy norm error estimate only first order estimates of the quadrature errors are needed but for $L^{2}$ error estimates second order estimates are necessary. To achieve a second order estimate of the quadrature error we utilize the fact that $\delta$ can be chosen in the form $\delta=C h$ with a sufficiently large $C$.

\subsection{Mapping the Exact Domain to the Approximate Domain}

The Mapping $\boldsymbol{F}_{\boldsymbol{h}}: \quad$ For $\delta>0$ let $\mathcal{U}_{\delta}(\Gamma)$ be the open tubular $\delta$ neighborhood

$$
\mathcal{U}_{\delta}(\Gamma)=\left\{x \in \mathbb{R}^{3}:|\rho(x)|<\delta\right\}
$$

For $0<\delta \leq \delta_{0}$, where $\delta_{0}$ is a constant, that only depend on the domain, chosen such that $\mathcal{U}_{\delta_{0}}(\Gamma) \subset \mathcal{U}(\Gamma)$, the mapping

$$
\mathcal{U}_{\delta}(\Gamma) \ni x \mapsto(p(x), \rho(x)) \in \Gamma \times(-\delta, \delta)
$$

is a bijection with inverse

$$
\Gamma \times(-\delta, \delta) \ni(x, z) \mapsto x+z n(x) \in \mathcal{U}_{\delta}(\Gamma)
$$

We next note that there is a function $\gamma_{h}: \Gamma \rightarrow \mathbb{R}$ such that

$$
q_{h}: \Gamma \ni x \mapsto x+n(x) \gamma_{h}(x) \in \Gamma_{h}
$$

is a bijection. Since for $x \in \Gamma_{h}$ there holds $p(x)=x-n^{e}(x) \rho(x)$ we may deduce that $q_{h}(x)$ is the inverse mapping to $p(x): \Gamma_{h} \mapsto \Gamma$. Using the assumptions on the approximation properties (3.2) and (3.3) we obtain the following estimates (see Appendix)

$$
\left\|\gamma_{h}\right\|_{L^{\infty}(\Gamma)} \lesssim h^{2}, \quad\left\|\nabla_{\Gamma} \gamma_{h}\right\|_{L^{\infty}(\Gamma)} \lesssim h
$$

Assuming that $h$ is sufficiently small so that $\Gamma_{h} \subset \mathcal{U}_{\delta / 3}(\Gamma)$ we may define the mapping

$$
F_{h}: \Omega_{0} \ni x \mapsto x+\chi(\rho(x)) n^{e}(x) \gamma_{h}^{e}(x) \in \Omega_{0}
$$

where $\chi:(-\delta, \delta) \rightarrow[0,1]$ is a smooth cut off function that equals 1 on $(-\delta / 3, \delta / 3)$ and 0 on $(-\delta, \delta) \backslash(-2 \delta / 3,2 \delta / 3)$ and the derivative $D \chi$ satisfies the estimate

$$
\|D \chi\|_{L^{\infty}(-\delta, \delta)} \lesssim \delta^{-1}
$$

We note that by construction $F_{h}: \Omega_{0} \rightarrow \Omega_{0}$ is a bijection such that

$$
F_{h}(\Omega)=\Omega_{h}, \quad F_{h}(\Gamma)=\Gamma_{h}
$$

and

$$
F_{h}=I \quad \text { in } \Omega_{0} \backslash \mathcal{U}_{\delta}(\Gamma)
$$


The Derivative $\boldsymbol{D} \boldsymbol{F}_{\boldsymbol{h}}$ : The derivative $D F_{h}(x) \in \mathcal{L}\left(\mathbb{R}^{3}, \mathbb{R}^{3}\right)$ of $F_{h}$ at $x \in \Omega_{0}$ is given by

$$
\begin{aligned}
D F_{h}(x)=I & +\left(\chi(\rho(x)) n^{e}(x)\right) D\left(\gamma_{h}^{e}(x)\right) \\
& +\left(D\left(\chi(\rho(x)) n^{e}(x)\right)\right) \gamma_{h}^{e}(x) \\
=I & +\left(\chi(\rho(x)) n^{e}(x)\right)\left(D \gamma_{h}\right)^{e}(x) D p(x) \\
& +\left((D \chi)(\rho(x)) D \rho(x) n^{e}(x)\right) \gamma_{h}^{e}(x) \\
& +\left(\chi(\rho(x))(D n)^{e}(x) D p(x)\right) \gamma_{h}^{e}(x)
\end{aligned}
$$

Next we note that

$$
D \rho=n^{e}, \quad D n=\mathcal{H}_{\Gamma}, \quad D p=P_{\Gamma}^{e}-\rho \mathcal{H}_{\Gamma}
$$

where we used the identity $p(x)=x-\rho(x) D \rho(x)=x-\rho(x) n^{e}(x)$ and introduced the curvature tensor $\mathcal{H}_{\Gamma}(x)=\nabla \otimes \nabla \rho(x), x \in \Gamma$. Note that it holds $\left.\left\|\mathcal{H}_{\Gamma}\right\|_{L^{\infty}\left(\mathcal{U}_{\delta}(\Gamma)\right.}\right) \lesssim 1$ for $\delta$ small enough. Thus we have

$$
\begin{aligned}
D F_{h}(x)=I & +\chi(\rho(x)) n^{e}(x) \otimes\left(\nabla_{\Gamma} \gamma_{h}\right)^{e}(x)\left(P_{\Gamma}^{e}(x)-\rho(x) \mathcal{H}_{\Gamma}(x)\right) \\
& +\gamma_{h}^{e}(x)(D \chi)(\rho(x)) n^{e}(x) \otimes n^{e}(x) \\
& +\chi(\rho(x)) \gamma_{h}^{e}(x) \mathcal{H}_{\Gamma}^{e}(x)\left(P_{\Gamma}^{e}(x)-\rho(x) \mathcal{H}_{\Gamma}(x)\right)
\end{aligned}
$$

On the surface $\Gamma$ we have the simplified expression

$$
D F_{h}(x)=I+n(x) \otimes \nabla_{\Gamma} \gamma_{h}(x)+\gamma_{h}(x) \mathcal{H}_{\Gamma}(x)
$$

since $\chi=1$ and $D \chi=0$ in a neighborhood of $\Gamma$ and $\rho(x)=0$ for $x \in \Gamma$. We note that $D F_{h}(x)$ maps the tangent space $T_{x}(\Gamma)$ into the piecewise defined tangent space $T_{F_{h}(x)}\left(\Gamma_{h}\right)$. In other words we have the identity

$$
D F_{h} P_{\Gamma}=\left(P_{\Gamma_{h}} \circ F_{h}\right) D F_{h} P_{\Gamma}
$$

and the mapping

$$
D F_{h, \Gamma}(x): T_{x}(\Gamma) \ni y \mapsto\left(P_{\Gamma_{h}} \circ F_{h}\right) D F_{h} P_{\Gamma} y \in T_{F_{h}(x)}\left(\Gamma_{h}\right)
$$

is invertible. Observing that by (4.5), $D F_{h}=I+O(h)$, for small enough $h$ we have the bounds

$$
\left\|D F_{h}\right\|_{L^{\infty}\left(\Omega_{0}, \mathcal{L}\left(\mathbb{R}^{3}, \mathbb{R}^{3}\right)\right)} \lesssim 1, \quad\left\|D F_{h}^{-1}\right\|_{L^{\infty}\left(\Omega_{0}, \mathcal{L}\left(\mathbb{R}^{3}, \mathbb{R}^{3}\right)\right)} \lesssim 1
$$

and

$$
\left\|D F_{h, \Gamma}\right\|_{L^{\infty}\left(\Gamma, \mathcal{L}\left(T_{x}(\Gamma), T_{F_{h}(x)}\left(\Gamma_{h}\right)\right)\right.} \lesssim 1, \quad\left\|D F_{h, \Gamma}^{-1}\right\|_{L^{\infty}\left(\Gamma, \mathcal{L}\left(T_{F_{h}(x)}\left(\Gamma_{h}\right), T_{x}(\Gamma)\right)\right.} \lesssim 1
$$

Below we simplify the notation as follows $\left\|D F_{h}\right\|_{L^{\infty}\left(\Omega_{0}\right)}=\left\|D F_{h}\right\|_{L^{\infty}\left(\Omega_{0}, \mathcal{L}\left(\mathbb{R}^{3}, \mathbb{R}^{3}\right)\right)}$ for the mappings $D F_{h}$ and $D F_{h, \Gamma}$ and their inverses. 
The Jacobian Determinants $\boldsymbol{J}_{\boldsymbol{h}}$ and $\boldsymbol{J}_{\boldsymbol{h}, \boldsymbol{\Gamma}}$ : We have the following relations between the measures on the exact and approximate surface and domain

$$
d \Omega_{h}=J F_{h} d \Omega, \quad d \Gamma_{h}=J F_{h, \Gamma} d \Gamma
$$

where the Jacobian determinants are defined by

$$
\begin{aligned}
J F_{h}(x) & =\left|\operatorname{det}\left(D F_{h}(x)\right)\right| \\
J F_{h, \Gamma}(x) & =\left|D F_{h, \Gamma}(x) \xi_{1} \times D F_{h, \Gamma}(x) \xi_{2}\right|
\end{aligned}
$$

and $\left\{\xi_{1}, \xi_{2}\right\}$ is an orthonormal basis in $T_{x}(\Gamma)$. We note that $J F_{h}=1$ on $\Omega_{0} \backslash \mathcal{U}_{\delta}(\Gamma)$ and recall that $D F_{h}=I+O(h)$. Thus we have the following estimates in the bulk

$$
\left\|J F_{h}\right\|_{L^{\infty}\left(\Omega_{0}\right)} \lesssim 1, \quad\left\|J F_{h}^{-1}\right\|_{L^{\infty}\left(\Omega_{0}\right)} \lesssim 1, \quad\left\|1-J F_{h}\right\|_{L^{\infty}\left(\mathcal{U}_{\delta}(\Gamma)\right)} \lesssim h
$$

since the determinant is a third order polynomial of the elements in $D F_{h}$. On the surface we note that

$$
D F_{h, \Gamma}(x) \xi=\xi+n\left(\xi \cdot \nabla_{\Gamma} \gamma_{h}\right)+\gamma_{h} \mathcal{H}_{\Gamma} \cdot \xi \quad \forall \xi \in T_{x}(\Gamma)
$$

where the last term is $O\left(h^{2}\right)$. The Jacobian determinant $J F_{h, \Gamma}$ is the norm of the cross product

$$
\begin{aligned}
\left|D F_{h, \Gamma}(x) \xi_{1} \times D F_{h, \Gamma}(x) \xi_{2}\right| & =\left|\left(\xi_{1}+n\left(\xi_{1} \cdot \nabla_{\Gamma} \gamma_{h}\right)\right) \times\left(\xi_{2}+n\left(\xi_{2} \cdot \nabla_{\Gamma} \gamma_{h}\right)\right)\right|+O\left(h^{2}\right) \\
& =\left|n-\xi_{1}\left(\xi_{1} \cdot \nabla_{\Gamma} \gamma_{h}\right)-\xi_{2}\left(\xi_{2} \cdot \nabla_{\Gamma} \gamma_{h}\right)\right|+O\left(h^{2}\right) \\
& =\left(1+\left(\xi_{1} \cdot \nabla_{\Gamma} \gamma_{h}\right)^{2}+\left(\xi_{2} \cdot \nabla_{\Gamma} \gamma_{h}\right)^{2}\right)^{1 / 2}+O\left(h^{2}\right) \\
& =1+O\left(h^{2}\right)
\end{aligned}
$$

where we used the identities $\xi_{1} \times \xi_{2}=n, n \times \xi_{2}=-\xi_{1}, \xi_{1} \times n=-\xi_{2}, n \times n=0$, the fact that $\left\{\xi_{1}, \xi_{2}, n\right\}$ is a positively oriented orthonormal basis in $\mathbb{R}^{3}$ to compute the norm, and finally the estimate $(1+\delta)^{1 / 2} \leq 1+\delta / 2, \delta>0$ in the last step. We thus have the following estimates for the surface Jacobian

$$
\left\|J F_{h, \Gamma}\right\|_{L^{\infty}(\Gamma)} \lesssim 1, \quad\left\|J F_{h, \Gamma}^{-1}\right\|_{L^{\infty}(\Gamma)} \lesssim 1, \quad\left\|1-J F_{h, \Gamma}\right\|_{L^{\infty}(\Gamma)} \lesssim h^{2}
$$

\subsection{Lifting to the Exact Domain}

We define the lifting or pullback of $v^{L}$ with respect to $F_{h}$ of a function $v$ defined on $\Omega_{0}$ as follows

$$
v^{L}:=v \circ F_{h}
$$

We note in particular that any function defined on $\Omega_{h}$ and $\Gamma_{h}$ may be lifted to a function on $\Omega$ and $\Gamma$. Using the chain rule

$$
D v^{L}=D\left(v \circ F_{h}\right)=\left(D v \circ F_{h}\right) D F_{h}=(D v)^{L} D F_{h}
$$


and thus we obtain the identities

$$
\begin{gathered}
\nabla v^{L}=D F_{h}^{T}\left(\nabla v \circ F_{h}\right)=D F_{h}^{T}(\nabla v)^{L} \\
\nabla_{\Gamma} v^{L}=P_{\Gamma} \nabla v^{L}=P_{\Gamma} D F_{h}^{T}(\nabla v)^{L} \\
=P_{\Gamma} D F_{h}^{T} P_{\Gamma_{h}}^{L}(\nabla v)^{L}=\left(P_{\Gamma} D F_{h}^{T} P_{\Gamma_{h}}^{L}\right)\left(\nabla_{\Gamma_{h}} v\right)^{L}=D F_{h, \Gamma}^{T}\left(\nabla_{\Gamma_{h}} v\right)^{L}
\end{gathered}
$$

where $D F_{h, \Gamma}$ was defined in (4.16). Summarizing, we have the relations

$$
\nabla v^{L}=D F_{h}^{T}(\nabla v)^{L}, \quad(\nabla v)^{L}=D F_{h}^{-T} \nabla v^{L}
$$

and

$$
\nabla_{\Gamma} v^{L}=D F_{h, \Gamma}^{T}\left(\nabla_{\Gamma_{h}} v\right)^{L}, \quad\left(\nabla_{\Gamma_{h}} v\right)^{L}=D F_{h, \Gamma}^{-T} \nabla_{\Gamma} v^{L}
$$

Using the bounds (4.17) and (4.18) we conclude that the following equivalences hold

$$
\left\|\nabla v^{L}\right\|_{L^{2}(\Omega)} \lesssim\left\|(\nabla v)^{L}\right\|_{L^{2}(\Omega)} \lesssim\left\|\nabla v^{L}\right\|_{L^{2}(\Omega)}
$$

and

$$
\left\|\nabla_{\Gamma} v^{L}\right\|_{L^{2}(\Gamma)} \lesssim\left\|\left(\nabla_{\Gamma_{h}} v\right)^{L}\right\|_{L^{2}(\Gamma)} \lesssim\left\|\nabla_{\Gamma} v^{L}\right\|_{L^{2}(\Gamma)}
$$

\subsection{Interpolation}

Let $E_{B}: H^{2}(\Omega) \rightarrow H^{2}\left(\Omega_{0}\right)$ be an extension operator such that

$$
\left\|E_{B} v\right\|_{H^{2}\left(\Omega_{0}\right)} \lesssim\|v\|_{H^{2}(\Omega)}
$$

and $E_{S}: H^{2}(\Gamma) \rightarrow H^{2}(\mathcal{U}(\Gamma))$ be the extension operator such that $E_{S} v=v \circ p$. Then we have the estimate

$$
\left\|E_{S} v\right\|_{H^{2}\left(\mathcal{U}_{\delta}(\Gamma)\right)} \lesssim \delta^{1 / 2}\|v\|_{H^{2}(\Gamma)}
$$

for any $\delta>0$ such that $\mathcal{U}_{\delta}(\Gamma) \subset \mathcal{U}(\Gamma)$. We finally define the extension operator

$$
E: H^{2}(\Omega) \times H^{2}(\Gamma) \ni\left(u_{B}, u_{S}\right) \mapsto\left(E_{B} u_{B}, E_{S} u_{S}\right) \in H^{2}\left(\Omega_{0}\right) \times H^{2}(\mathcal{U}(\Gamma))
$$

When suitable we simplify the notation and write $u=E u$. We let $\pi_{S Z, h}: L^{2}\left(\Omega_{0}\right) \rightarrow V_{0, h}$ denote the standard Scott-Zhang interpolation operator and recall the interpolation error estimate

$$
\left\|v-\pi_{S Z, h} v\right\|_{H^{m}(K)} \leq C h^{2-m}\|v\|_{H^{2}(\mathcal{N}(K))}, \quad m=1,2, \quad K \in \mathcal{K}_{0, h}
$$

where $\mathcal{N}(K) \subset \Omega_{h}$ is the union of the neighboring elements of $K$. We then define the interpolant

$$
\pi_{h} u=\left(\pi_{B, h} u_{B}, \pi_{S, h} u_{S}\right)
$$

where

$$
\pi_{B, h} u_{B}=\left.\left(\pi_{S Z, h} E_{B} u_{B}\right)\right|_{\mathcal{N}_{B, h}} \in V_{B, h}
$$


and

$$
\pi_{S, h} u_{S}=\left.\left(\pi_{S Z, h} E_{S} u_{S}\right)\right|_{\mathcal{N}_{S, h}} \in V_{S, h}
$$

We use the notation

$$
\pi_{h}^{L} u=\left(\pi_{h} u\right)^{L}=\left(\pi_{h} u\right) \circ F_{h}
$$

for the pullback of $\pi_{h} u$ to $\Omega$ by $F_{h}$. With these definitions we have the following lemma:

Lemma 4.1 The following estimate holds

$$
\left\|u-\pi_{h}^{L} u\right\| \mid \lesssim h\|u\|_{H^{2}(\Omega) \times H^{2}(\Gamma)}
$$

Proof. Using a trace inequality we obtain

$$
\begin{aligned}
\left\|u-\pi_{h}^{L} u\right\| \|^{2}= & b_{B} k_{B}\left\|\nabla\left(u_{B}-\pi_{B, h}^{L} u_{B}\right)\right\|_{L^{2}(\Omega)}^{2}+b_{S} k_{S}\left\|\nabla\left(u_{S}-\pi_{S, h}^{L} u_{S}\right)\right\|_{L^{2}(\Gamma)}^{2} \\
& \quad+\left\|b_{B}\left(u_{B}-\pi_{B, h}^{L} u_{B}\right)-b_{S}\left(u_{S}-\pi_{S, h}^{L} u_{S}\right)\right\|_{L^{2}(\Gamma)}^{2} \\
\lesssim & \left\|u_{B}-\pi_{B, h}^{L} u_{B}\right\|_{H^{1}(\Omega)}^{2}+\left\|u_{S}-\pi_{S, h}^{L} u_{S}\right\|_{H^{1}(\Gamma)}^{2} \\
& =I+I I
\end{aligned}
$$

Term $\boldsymbol{I}$. The first term may be estimated as follows

$$
\begin{aligned}
I & =\left\|u_{B}-\pi_{B, h}^{L} u_{B}\right\|_{H^{1}(\Omega)}=\left\|u_{B}-\left(\left.\pi_{S Z, h} E_{B} u_{B}\right|_{\Omega_{h}}\right)^{L}\right\|_{H^{1}(\Omega)} \\
& \leq\left\|u_{B}-\left(\left.E_{B} u_{B}\right|_{\Omega_{h}}\right)^{L}\right\|_{H^{1}(\Omega)}+\left\|\left(\left.\left(I-\pi_{S Z, h}\right) E_{B} u_{B}\right|_{\Omega_{h}}\right)^{L}\right\|_{H^{1}(\Omega)} \\
& \lesssim h\left\|u_{B}\right\|_{H^{2}(\Omega)}
\end{aligned}
$$

Here we used the Sobolev Taylor's formula, see [2], to estimate the first term: consider first a function $v \in H^{2}\left(\Omega_{0}\right)$; then we have

$$
\left\|v-v \circ F_{h}\right\|_{L^{2}\left(\Omega_{0}\right)} \lesssim\left\|I-F_{h}\right\|_{L^{\infty}\left(\Omega_{0}\right)}\|\nabla v\|_{L^{2}\left(\Omega_{0}\right)} \lesssim h^{2}\|v\|_{H^{1}\left(\Omega_{0}\right)}
$$

and for the derivative

$$
\begin{aligned}
\| \nabla(v & \left.-v \circ F_{h}\right) \|_{L^{2}\left(\Omega_{0}\right)} \\
& =\left\|\nabla v-D F_{h}^{T}\left(\nabla v \circ F_{h}\right)\right\|_{L^{2}\left(\Omega_{0}\right)} \\
& \leq\left\|\nabla v-\left(\nabla v \circ F_{h}\right)\right\|_{L^{2}\left(\Omega_{0}\right)}+\left\|\left(D F_{h}^{T}-I\right)\left(\nabla v \circ F_{h}\right)\right\|_{L^{2}\left(\Omega_{0}\right)} \\
& \lesssim\left\|I-F_{h}\right\|_{L^{\infty}\left(\Omega_{0}\right)}\|\nabla v\|_{H^{1}\left(\Omega_{0}\right)}+\left\|\left(D F_{h}^{T}-I\right)\right\|_{L^{\infty}\left(\Omega_{0}\right)}\|\nabla v\|_{L^{2}\left(\Omega_{0}\right)} \\
& \lesssim h^{2}\|v\|_{H^{2}\left(\Omega_{0}\right)}+h\|\nabla v\|_{L^{2}\left(\Omega_{0}\right)} \\
& \lesssim h\|v\|_{H^{2}\left(\Omega_{0}\right)}
\end{aligned}
$$

Now we may apply these inequalities with $v=E_{B} u_{B}$ and finally use the stability (4.34) of the extension operator $E_{B}$.

The second term in (4.44) is estimated by mapping to the discrete domain using the interpolation estimate (4.37) and then using the stability estimate (4.34). 
Term II. Changing domain of integration from $\Gamma$ to $\Gamma_{h}$ and then using an element--wise trace inequality we obtain

$$
\begin{aligned}
\left\|\nabla_{\Gamma}\left(u_{S}-\pi_{S, h}^{L} u_{S}\right)\right\|_{L^{2}(\Gamma)}^{2} & =\left\|D F_{h, \Gamma}^{T} \nabla_{\Gamma_{h}}\left(u_{S}^{e}-\pi_{S, h} u_{S}\right)\left|J F_{h, \Gamma}\right|^{-1 / 2}\right\|_{L^{2}\left(\Gamma_{h}\right)}^{2} \\
& \lesssim \sum_{K \in \mathcal{K}_{S, h}} h^{-1}\left\|u_{S}^{e}-\pi_{S, h} u_{S}\right\|_{H^{1}(K)}+h\left\|u_{S}^{e}-\pi_{S, h} u_{S}\right\|_{H^{2}(K)}^{2} \\
& \lesssim \sum_{K \in \mathcal{K}_{S, h}} h\left\|u_{S}^{e}\right\|_{H^{2}(\mathcal{N}(K))}^{2} \\
& \lesssim h^{2}\left\|u_{S}\right\|_{H^{2}(\Gamma)}^{2}
\end{aligned}
$$

Here we used the interpolation estimate (4.37) followed by the stability estimate 4.35) for the extension operator with $\delta \sim h$, which is possible since there is $\delta \lesssim h$ such that $\mathcal{K}_{S, h} \subset \mathcal{U}_{\delta}(\Gamma)$

We also need the face norm

$$
\begin{aligned}
\|v\|_{\mathcal{F}}^{2} & =h^{3} j_{B}\left(v_{B, h}, v_{B, h}\right)+j_{S}\left(v_{S, h}, v_{S, h}\right) \\
& =\sum_{F \in \mathcal{F}_{B, h}} h^{3}\left\|\left[n_{F} \cdot \nabla v_{B}\right]\right\|_{L^{2}(F)}^{2}+\sum_{F \in \mathcal{F}_{S, h}} \|\left[n_{F} \cdot \nabla v_{S} \|_{L^{2}(F)}^{2}\right.
\end{aligned}
$$

for which we have the following interpolation error estimate.

Lemma 4.2 The following estimate holds

$$
\left\|u-\pi_{h} u\right\|_{\mathcal{F}} \lesssim h\|u\|_{H^{2}(\Omega) \times H^{2}(\Gamma)}
$$

Proof. This estimate follows directly by using an element wise trace inequality, followed by the interpolation estimate (4.37), and finally the stability estimates 4.34 and 4.35) for the extension operators.

\subsection{Strang's Lemma}

Lemma 4.3 The following estimate holds

$$
\begin{aligned}
\left(\|\| u-u_{h}^{L}||^{2}+\| u-u_{h}||_{\mathcal{F}}^{2}\right)^{1 / 2} \lesssim( & \left.\left.\left\|u-\pi_{h}^{L} u\right\|\right|^{2}+\left\|u-\pi_{h} u\right\|_{\mathcal{F}}^{2}\right)^{1 / 2} \\
& +\sup _{v \in W_{h}} \frac{a\left(u_{h}^{L}, v^{L}\right)-a_{h}\left(u_{h}, v\right)}{\left\|v^{L}\right\| \mid} \\
& +\sup _{v \in W_{h}} \frac{l\left(v^{L}\right)-l_{h}(v)}{\left\|v^{L}\right\|}
\end{aligned}
$$


Proof. Adding and subtracting an interpolant $\pi_{h}^{L} u$, defined by (4.41), and using the triangle inequality we obtain

$$
\begin{aligned}
\left(\|\| u-\left.u_{h}^{L}\left|\left\|^{2}+\right\| \| u-u_{h}\right|\right|_{\mathcal{F}} ^{2}\right)^{1 / 2} \leq & \left(\left\|u-\pi_{h}^{L} u\left|\left\|_{h}^{2}+\right\|\left\|u-\pi_{h} u \mid\right\|_{\mathcal{F}}^{2}\right)^{1 / 2}\right.\right. \\
& +\left(\left\|\pi_{h}^{L} u-u_{h}^{L}\right\|_{h}+\|\| \pi_{h} u-u_{h} \mid \|_{\mathcal{F}}^{2}\right)^{1 / 2}
\end{aligned}
$$

To estimate the second term we start from the coercivity

$$
\left(\left|\left\|\pi_{h}^{L} u-u_{h}^{L}||^{2}+\right\|\right| \pi_{h} u-u_{h}||_{\mathcal{F}}^{2}\right)^{1 / 2} \leq \sup _{v \in W_{h} \backslash\{0\}} \frac{a\left(\pi_{h}^{L} u-u_{h}^{L}, v^{L}\right)+j_{h}\left(\pi_{h} u-u_{h}, v\right)}{\left(\left\|v^{L}||^{2}+\right\| v \|\left.\right|_{\mathcal{F}} ^{2}\right)^{1 / 2}}
$$

Adding and subtracting the exact solution, and using Galerkin orthogonality the numerator may be written in the following form

$$
\begin{aligned}
a\left(\pi_{h}^{L} u-u_{h}^{L}, v^{L}\right)+j_{h}\left(\pi_{h} u-u_{h}, v\right) \\
=a\left(\pi_{h}^{L} u-u, v^{L}\right)+a\left(u-u_{h}^{L}, v^{L}\right)+j_{h}\left(\pi_{h} u-u_{h}, v\right) \\
=a\left(\pi_{h}^{L} u-u, v^{L}\right)+l\left(v^{L}\right)-a\left(u_{h}^{L}, v^{L}\right)+j_{h}\left(\pi_{h} u-u_{h}, v\right) \\
=a\left(\pi_{h}^{L} u-u, v^{L}\right)+l\left(v^{L}\right)-l_{h}(v) \\
\quad \quad+a_{h}\left(u_{h}, v\right)+j_{h}\left(u_{h}, v\right)-a\left(u_{h}^{L}, v^{L}\right)+j_{h}\left(\pi_{h} u-u_{h}, v\right) \\
=a\left(\pi_{h}^{L} u-u, v^{L}\right)+j_{h}\left(\pi_{h} u-u, v\right) \\
\quad+\left(a_{h}\left(u_{h}, v\right)-a\left(u_{h}^{L}, v^{L}\right)\right)+\left(l\left(v^{L}\right)-l_{h}(v)\right)
\end{aligned}
$$

Using 4.53) and estimating the first term using the Cauchy-Schwarz inequality the lemma follows directly.

\subsection{Estimate of the Quadrature Errors}

Lemma 4.4 If $h \lesssim \delta \leq \delta_{0}$ and $h$ is small enough. Then it holds

$$
\begin{aligned}
\left|a\left(v^{L}, w^{L}\right)-a_{h}(v, w)\right| \lesssim & h^{2}\left\|\nabla_{\Gamma} v_{S}^{L}\right\|_{L^{2}(\Gamma)}\left\|\nabla_{\Gamma} w_{S}^{L}\right\|_{L^{2}(\Gamma)} \\
& +h^{2}\left\|b \cdot v^{L}\right\|_{L^{2}(\Gamma)}\left\|b \cdot w^{L}\right\|_{L^{2}(\Gamma)} \\
& +h\left\|\nabla v_{B}^{L}\right\|_{L^{2}\left(\mathcal{U}_{\delta}(\Gamma) \cap \Omega\right)}\left\|\nabla w_{B}^{L}\right\|_{L^{2}\left(\mathcal{U}_{\delta}(\Gamma) \cap \Omega\right)} \quad \forall v, w \in W_{h}
\end{aligned}
$$

Proof. Using the definition of the bilinear forms we have

$$
\begin{aligned}
a\left(v^{L}, w^{L}\right)-a_{h}(v, w)= & \underbrace{a_{B}\left(v_{B}^{L}, w_{B}^{L}\right)-a_{B, h}\left(v_{B}, w_{B}\right)}_{I} \\
& +\underbrace{a_{S}\left(v_{S}^{L}, w_{S}^{L}\right)-a_{S, h}\left(v_{S}, w_{S}\right)}_{I I}+\underbrace{a_{B S}(v, w)-a_{B S, h}(v, w)}_{I I I} \\
= & I+I I+I I I
\end{aligned}
$$


We now proceed with estimates of the three terms.

Term I. Starting from the definition of the forms (2.15) and (3.10), changing domain of integration to $\Omega$, and using (4.30), we obtain the following identity

$$
\begin{aligned}
&\left(b_{B} k_{B}\right)^{-1}\left(a_{B}\left(v_{B}^{L}, w_{B}^{L}\right)-a_{B, h}\left(v_{B}, w_{B}\right)\right)=\left(D F_{h}^{T}\left(\nabla v_{B}\right)^{L}, D F_{h}^{T}\left(\nabla w_{B}\right)^{L}\right)_{\Omega}-\left(\nabla v_{B}, \nabla w_{B}\right)_{\Omega_{h}} \\
& \quad=\left(D F_{h}^{T}\left(\nabla v_{B}\right)^{L}, D F_{h}^{T}\left(\nabla w_{B}\right)^{L}\right)_{\Omega}-\left(\left(\nabla v_{B}\right)^{L},\left(\nabla w_{B}\right)^{L} J F_{h}\right)_{\Omega} \\
& \quad=\left(\left(D F_{h} D F_{h}^{T}-J F_{h} I\right)\left(\nabla v_{B}\right)^{L},\left(\nabla w_{B}\right)^{L}\right)_{\Omega} \\
& \quad=\left(\mathcal{A}_{h, \Omega}\left(\nabla v_{B}\right)^{L},\left(\nabla w_{B}\right)^{L}\right)_{\Omega}
\end{aligned}
$$

In order to estimate $\mathcal{A}_{h, \Omega}=D F_{h} D F_{h}^{T}-J F_{h} I$ we note that $\mathcal{A}_{h, \Omega}=0$ in $\Omega_{0} \backslash \mathcal{U}_{\delta}(\Gamma)$ and in $\mathcal{U}_{\delta}(\Gamma)$ we have the identity

$$
\begin{aligned}
\mathcal{A}_{h, \Omega} & =D F_{h} D F_{h}^{T}-J F_{h} I \\
& =\left(D F_{h}-I\right)\left(D F_{h}-I\right)^{T}+\left(D F_{h}+D F_{h}^{T}\right)-I-J F_{h} I \\
& =\left(D F_{h}-I\right)\left(D F_{h}-I\right)^{T}+\left(D F_{h}-I\right)+\left(D F_{h}-I\right)^{T}+\left(1-J F_{h}\right) I
\end{aligned}
$$

and therefore we have the estimate

$$
\begin{aligned}
\left\|\mathcal{A}_{h, \Omega}\right\|_{L^{\infty}\left(\mathcal{U}_{\delta}(\Gamma) \cap \Omega\right)} \lesssim & \left\|D F_{h}-I\right\|_{L^{\infty}\left(\mathcal{U}_{\delta}(\Gamma) \cap \Omega\right)}^{2} \\
& +\left\|D F_{h}-I\right\|_{L^{\infty}\left(\mathcal{U}_{\delta}(\Gamma) \cap \Omega\right)}+\left\|1-J F_{h}\right\|_{L^{\infty}\left(\mathcal{U}_{\delta}(\Gamma) \cap \Omega\right)}
\end{aligned}
$$

This estimate holds for any $0<\delta \leq \delta_{0}$ and $h$ such that

$$
\Gamma_{h} \subset \mathcal{U}_{\delta / 3}(\Gamma)
$$

Recall that (4.60) is required in the definition (4.6) of the mapping $F_{h}$. Now using the assumption that there is a constant $C_{1}>0$ such that $C_{1} h<\delta \leq \delta_{0}$, there is a constant $h_{0}>0$, independent of $\delta$, such that (4.60) holds for $0<h \leq h_{0}$, since we have the estimate $\left\|\gamma_{h}\right\|_{L^{\infty}(\Gamma)} \leq C_{2} h^{2} \leq\left(C_{2} h_{0}\right) h<C_{1} h / 3<\delta / 3$, where we may choose $h_{0}$ such that $C_{2} h_{0}<C_{1} / 3$.

Proceeding with the estimate of $\left\|D F_{h}-I\right\|_{L^{\infty}\left(\mathcal{U}_{\delta}(\Gamma) \cap \Omega\right)}$ for $C_{1} h<\delta \leq \delta_{0}$ and $0<h \leq h_{0}$ we start from the identity (4.13) and then using the estimates $\|\chi\|_{L^{\infty}(-\delta, \delta)}=1,0<\delta \leq \delta_{0}$ and $\left\|P_{\Gamma}^{e}-\rho \mathcal{H}_{\Gamma}\right\|_{L^{\infty}\left(\mathcal{U}_{\delta_{0}}(\Gamma)\right)} \lesssim 1$ we obtain

$$
\begin{aligned}
\left\|D F_{h}-I\right\|_{L^{\infty}\left(\mathcal{U}_{\delta}(\Gamma) \cap \Omega\right)} \lesssim & \left\|\nabla_{\Gamma} \gamma_{h}\right\|_{L^{\infty}(\Gamma)} \\
& \quad+\left\|\gamma_{h}\right\|_{L^{\infty}(\Gamma)}\|D \chi\|_{L^{\infty}(-\delta, \delta)}+\left\|\gamma_{h}\right\|_{L^{\infty}(\Gamma)} \\
& \lesssim h+h^{2} \delta^{-1}+h^{2} \\
\lesssim & \lesssim h
\end{aligned}
$$


where we used (4.5) and (4.7) and $C_{1} h<\delta$. This estimate holds for all $\delta$ and $h$ such that $C_{1} h<\delta \leq \delta_{0}$ and $0<h \leq h_{0}$. Combining (4.61) with the estimate for the Jacobian determinant 4.22 we obtain the estimate

$$
\left\|\mathcal{A}_{h, \Omega}\right\|_{L^{\infty}\left(\mathcal{U}_{\delta}(\Gamma) \cap \Omega\right)} \lesssim h
$$

and we also recall that

$$
\mathcal{A}_{h, \Omega}=0 \quad \text { in } \Omega \backslash \mathcal{U}_{\delta}(\Gamma)
$$

Using the bound 4.62 for $\mathcal{A}_{h, \Omega}$ we obtain the estimate

$$
\begin{aligned}
\left|a_{B}\left(v^{L}, w^{L}\right)-a_{B, h}(v, w)\right| & \lesssim h\left\|(\nabla v)^{L}\right\|_{L^{2}\left(\mathcal{U}_{\delta}(\Gamma)\right)}\left\|(\nabla w)^{L}\right\|_{L^{2}\left(\mathcal{U}_{\delta}(\Gamma)\right)} \\
& \lesssim h\left\|\nabla v^{L}\right\|_{L^{2}\left(\mathcal{U}_{\delta}(\Gamma)\right)}\left\|\nabla w^{L}\right\|_{L^{2}\left(\mathcal{U}_{\delta}(\Gamma)\right)}
\end{aligned}
$$

At last we used the estimate

$$
\begin{aligned}
\left\|(\nabla v)^{L}\right\|_{L^{2}\left(\mathcal{U}_{\delta}(\Gamma)\right)} & =\left\|D F_{h}^{-T}\left(\nabla v^{L}\right)\right\|_{L^{2}\left(\mathcal{U}_{\delta}(\Gamma)\right)} \\
& =\left\|D F_{h}^{-T}\right\|_{L^{\infty}\left(\mathcal{U}_{\delta}(\Gamma)\right)}\left\|\nabla v^{L}\right\|_{L^{2}\left(\mathcal{U}_{\delta}(\Gamma)\right)} \lesssim\left\|\nabla v^{L}\right\|_{L^{2}\left(\mathcal{U}_{\delta}(\Gamma)\right)}
\end{aligned}
$$

where we employed 4.17).

Term II. Proceeding in the same way and using (4.31) we obtain

$$
\begin{aligned}
\left(b_{S} k_{S}\right)^{-1} & \left(a_{S}\left(v_{S}^{L}, w_{S}^{L}\right)-a_{S, h}\left(v_{S}, w_{S}\right)\right) \\
& =\left(\nabla_{\Gamma} v_{S}^{L}, \nabla_{\Gamma} w_{S}^{L}\right)_{\Gamma}-\left(\nabla_{\Gamma_{h}} v_{S}, \nabla_{\Gamma_{h}} w_{S}\right)_{\Gamma_{h}} \\
& =\left(D F_{h, \Gamma}^{T}\left(\nabla_{\Gamma_{h}} v_{S}\right)^{L}, D F_{h, \Gamma}^{T}\left(\nabla_{\Gamma_{h}} w_{S}\right)^{L}\right)_{\Gamma}-\left(\left(\nabla_{\Gamma_{h}} v_{S}\right)^{L},\left(\nabla_{\Gamma_{h}} w_{S}\right)^{L} J F_{h, \Gamma}\right)_{\Gamma} \\
& =\left(\left(D F_{h, \Gamma} D F_{h, \Gamma}^{T}-P_{\Gamma_{h}}^{L} J F_{h, \Gamma}\right)\left(\nabla_{\Gamma_{h}} v_{S}\right)^{L},\left(\nabla_{\Gamma_{h}} w_{S}\right)^{L}\right)_{\Gamma} \\
& =\left(\mathcal{A}_{\Gamma, h}\left(\nabla_{\Gamma_{h}} v_{S}\right)^{L},\left(\nabla_{\Gamma_{h}} w_{S}\right)^{L}\right)_{\Gamma}
\end{aligned}
$$

where we introduced

$$
\mathcal{A}_{\Gamma, h}=D F_{h, \Gamma} D F_{h, \Gamma}^{T}-P_{\Gamma_{h}}^{L} J F_{h, \Gamma}
$$

Using the definition (4.16) of $D F_{h, \Gamma}$ and the expression 4.14 for $D F_{h}$ we have the identity

$$
\begin{aligned}
D F_{h, \Gamma} & =P_{\Gamma_{h}}^{L} D F_{h} P_{\Gamma} \\
& =P_{\Gamma_{h}}^{L}\left(I+n \otimes \nabla_{\Gamma} \gamma_{h}+\gamma_{h} \mathcal{H}_{\Gamma}\right) P_{\Gamma} \\
& =P_{\Gamma_{h}}^{L} P_{\Gamma}+\left(P_{\Gamma_{h}}^{L} n\right) \otimes \nabla_{\Gamma} \gamma_{h}+\gamma_{h} P_{\Gamma_{h}}^{L} \mathcal{H}_{\Gamma} P_{\Gamma}
\end{aligned}
$$

Here the second term can be estimated as follows

$$
\left\|\left(P_{\Gamma_{h}}^{L} n\right) \otimes \nabla_{\Gamma} \gamma_{h}\right\|_{L^{\infty}(\Gamma)} \lesssim\left\|P_{\Gamma_{h}}^{L} n\right\|_{L^{\infty}(\Gamma)}\left\|\nabla_{\Gamma} \gamma_{h}\right\|_{L^{\infty}(\Gamma)} \lesssim h^{2}
$$


where we used the estimate

$$
\left\|P_{\Gamma_{h}}^{L} n\right\|_{L^{\infty}(\Gamma)}=\left\|P_{\Gamma_{h}}^{L}\left(n-n_{h}^{L}\right)\right\|_{L^{\infty}(\Gamma)} \lesssim\left\|n \circ p-n_{h}\right\|_{L^{\infty}\left(\Gamma_{h}\right)} \lesssim h
$$

For the third term we have the estimate

$$
\left\|\gamma_{h} P_{\Gamma_{h}}^{L} \mathcal{H}_{\Gamma} P_{\Gamma}\right\|_{L^{\infty}(\Gamma)} \lesssim\left\|\gamma_{h}\right\|_{L^{\infty}(\Gamma)}\left\|P_{\Gamma_{h}}^{L}\right\|_{L^{\infty}(\Gamma)}\left\|\mathcal{H}_{\Gamma}\right\|_{L^{\infty}(\Gamma)}\left\|P_{\Gamma}\right\|_{L^{\infty}(\Gamma)} \lesssim h^{2}
$$

Thus we conclude that

$$
D F_{h, \Gamma}=P_{\Gamma_{h}}^{L} P_{\Gamma}+O\left(h^{2}\right)
$$

Inserting this identity into the expression (4.67) for $\mathcal{A}_{\Gamma, h}$ and using the identity

$$
P_{\Gamma_{h}}^{L} J F_{h, \Gamma}=P_{\Gamma_{h}}^{L}+P_{\Gamma_{h}}^{L}\left(J F_{h, \Gamma}-1\right)=P_{\Gamma_{h}}^{L}+O\left(h^{2}\right)
$$

where we used (4.25), we obtain

$$
\mathcal{A}_{\Gamma, h}=P_{\Gamma_{h}}^{L} P_{\Gamma} P_{\Gamma_{h}}^{L}-P_{\Gamma_{h}}^{L}+O\left(h^{2}\right)
$$

Now the following identity holds

$$
P_{\Gamma_{h}}^{L} P_{\Gamma} P_{\Gamma_{h}}^{L}-P_{\Gamma_{h}}^{L}=P_{\Gamma_{h}}^{L}\left(P_{\Gamma}-P_{\Gamma_{h}}^{L}\right)\left(P_{\Gamma}-P_{\Gamma_{h}}^{L}\right) P_{\Gamma_{h}}^{L}
$$

which leads to the estimate

$$
\left\|P_{\Gamma_{h}}^{L} P_{\Gamma} P_{\Gamma_{h}}^{L}-P_{\Gamma_{h}}^{L}\right\|_{L^{\infty}(\Gamma)} \leq\left\|P_{\Gamma_{h}}^{L}\right\|_{L^{\infty}(\Gamma)}^{2}\left\|P_{\Gamma}-P_{\Gamma_{h}}^{L}\right\|_{L^{\infty}(\Gamma)}^{2} \lesssim h^{2}
$$

where we used the bound

$$
\begin{aligned}
\left\|P_{\Gamma}-P_{\Gamma_{h}}^{L}\right\|_{L^{\infty}(\Gamma)} & =\left\|n \otimes n-n_{h}^{L} \otimes n_{h}^{L}\right\|_{L^{\infty}(\Gamma)} \\
& \lesssim\left\|\left(n-n_{h}^{L}\right) \otimes n\right\|_{L^{\infty}(\Gamma)}+\left\|n_{h}^{L} \otimes\left(n-n_{h}^{L}\right)\right\|_{L^{\infty}(\Gamma)} \\
& \lesssim\left\|n^{e}-n_{h}\right\|_{L^{\infty}\left(\Gamma_{h}\right)} \\
& \lesssim h
\end{aligned}
$$

Thus we finally arrive at

$$
\left\|\mathcal{A}_{\Gamma, h}\right\|_{L^{\infty}(\Gamma)} \lesssim h^{2}
$$

and therefore we have the estimate

$$
\begin{aligned}
\left|a_{S}\left(v^{L}, w^{L}\right)-a_{S, h}(v, w)\right| & \lesssim h^{2}\left\|\left(\nabla_{\Gamma_{h}} v\right)^{L}\right\|_{L^{2}(\Gamma)}\left\|\left(\nabla_{\Gamma_{h}} w\right)^{L}\right\|_{L^{2}(\Gamma)} \\
& \lesssim h^{2}\left\|\nabla_{\Gamma} v^{L}\right\|_{L^{2}(\Gamma)}\left\|\nabla_{\Gamma} w^{L}\right\|_{L^{2}(\Gamma)}
\end{aligned}
$$

where at last we used 4.18. 
Term III. We have

$$
\begin{aligned}
a_{B S}\left(v^{L}, w^{L}\right)-a_{B S, h}(v, w) & =\left(b \cdot v^{L}, b \cdot w^{L}\right)_{\Gamma}-(b \cdot v, b \cdot w)_{\Gamma_{h}} \\
& =\left(\left(1-J F_{\Gamma, h}\right) b \cdot v^{L}, b \cdot w^{L}\right)_{\Gamma}
\end{aligned}
$$

and thus we obtain the estimate

$$
\left|a_{B S}\left(v^{L}, w^{L}\right)-a_{B S, h}(v, w)\right| \lesssim h^{2}\left\|b \cdot v^{L}\right\|_{L^{2}(\Gamma)}\left\|b \cdot w^{L}\right\|_{L^{2}(\Gamma)}
$$

Lemma 4.5 If $f_{h}=\left(f_{B, h}, f_{S, h}\right)$ satisfies the estimate

$$
\left\|f_{B}-f_{B, h}^{L}\right\|_{L^{2}(\Omega)}+\left\|f_{S}-f_{S, h}^{L}\right\|_{L^{2}(\Gamma)} \lesssim h^{2}
$$

Then it holds

$$
\left|l\left(v^{L}\right)-l_{h}(v)\right| \lesssim h^{2}\left\|v^{L}\right\|_{L^{2}(\Omega) \times L^{2}(\Gamma)} \quad \forall v \in W_{h}
$$

Proof. We have

$$
\begin{aligned}
l\left(v^{L}\right)-l_{h}(v) & =b_{B}\left(f_{B}, v_{B}^{L}\right)_{\Omega}-b_{B}\left(f_{B, h}, v_{B}\right)_{\Omega_{h}}+b_{S}\left(f_{S}, v_{S}^{L}\right)_{\Gamma}-b_{S}\left(f_{S, h}, v_{S}\right)_{\Gamma_{h}} \\
& =b_{B}\left(f_{B}-f_{B, h}^{L} J F_{h}, v_{B}^{L}\right)_{\Omega}+b_{S}\left(f_{S}-f_{S, h}^{L} J F_{h}, v_{S}^{L}\right)_{\Gamma}
\end{aligned}
$$

which immediately leads to the estimate

$$
\left|l\left(v^{L}\right)-l_{h}(v)\right| \lesssim h^{2}\left\|v^{L}\right\|_{L^{2}(\Omega) \times L^{2}(\Gamma)}
$$

\subsection{Error Estimates}

Theorem 4.1 The following error estimate holds

$$
\left(\left\|u-u_{h}^{L} \mid\right\|^{2}+\left\|u-u_{h}\right\|_{\mathcal{F}}^{2}\right)^{1 / 2} \lesssim h\|u\|_{H^{2}(\Omega) \times H^{2}(\Gamma)}
$$

for small enough mesh parameter $h$.

Proof. Using the Strang Lemma, Lemma 4.3, in combination with the quadrature error estimates in Lemma 4.4 and 4.5 , we obtain

$$
\begin{aligned}
\left(\|\| u-u_{h}^{L}\left\|^{2}+\right\| u-u_{h} \|_{\mathcal{F}}^{2}\right)^{1 / 2} \lesssim & \left(\left.\left\|u-\pi_{h}^{L} u\right\|\right|^{2}+\left\|u-\pi_{h} u\right\|_{\mathcal{F}}^{2}\right)^{1 / 2} \\
& +\sup _{v \in W_{h}} \frac{a\left(u_{h}^{L}, v^{L}\right)-a_{h}\left(u_{h}, v\right)}{\left\|v^{L} \mid\right\|}+\sup _{v \in W_{h}} \frac{l\left(v^{L}\right)-l_{h}(v)}{\left\|v^{L}\right\|} \\
\lesssim & \left(\left.\left\|u-\pi_{h}^{L} u\right\|\right|^{2}+\left\|u-\pi_{h} u\right\|_{\mathcal{F}}^{2}\right)^{1 / 2}+h\left\|u_{h}^{L}\right\| \|+h^{2} \\
\lesssim & h
\end{aligned}
$$


Here we used the interpolation error estimates in Lemma 4.1 and Lemma 4.2, and the stability estimate

$$
\left\|\left|u_{h}^{L}\right|\right\| \lesssim\|f\|_{L^{2}(\Omega) \times L^{2}(\Gamma)}
$$

in the last inequality.

Theorem 4.2 The following error estimate holds

$$
\left\|u-u_{h}^{L}\right\|_{L^{2}(\Omega) \times L^{2}(\Gamma)} \lesssim h^{2}\|u\|_{H^{2}(\Omega) \times H^{2}(\Gamma)}
$$

for small enough mesh parameter $h$.

Proof. Let $\phi$ be the solution to the dual problem: find $\phi \in W$ such that

$$
a(v, \phi)=(v, \psi)_{L^{2}(\Omega) \times L^{2}(\Gamma)} \quad \forall v \in W
$$

where $\psi=\left(\psi_{B}, \psi_{S}\right) \in L^{2}(\Omega) \times L^{2}(\Gamma)$. Then we have the regularity estimate

$$
\|\phi\|_{H^{2}(\Omega) \times H^{2}(\Gamma)} \lesssim\|\psi\|_{L^{2}(\Omega) \times L^{2}(\Gamma)}
$$

Setting $v=u-u_{h}^{L}$, and adding and subtracting suitable terms we obtain

$$
\begin{aligned}
\left(u_{B}-u_{B, h}^{L}, \psi_{B}\right)_{\Omega}+\left(u_{S}-u_{S, h}^{L}, \psi_{S}\right)_{\Gamma}= & a\left(u-u_{h}^{L}, \phi\right) \\
= & a\left(u-u_{h}^{L}, \phi-\pi_{h}^{L} \phi\right)+a\left(u-u_{h}^{L}, \pi_{h}^{L} \phi\right) \\
= & a \underbrace{a\left(u-u_{h}^{L}, \phi-\pi_{h}^{L} \phi\right)}_{I}+\underbrace{\left(l\left(\pi_{h}^{L} \phi\right)-l_{h}\left(\pi_{h} \phi\right)\right)}_{I I} \\
& +\underbrace{\left(a_{h}\left(u_{h}, \pi_{h} \phi\right)-a\left(u_{h}^{L}, \pi_{h}^{L} \phi\right)\right)}_{I I I}+\underbrace{j_{h}\left(u_{h}, \pi_{h} \phi\right)}_{I V} \\
= & I+I I+I I I+I V
\end{aligned}
$$

Term I. Using Cauchy-Schwarz, the energy norm estimate 4.85), the interpolation estimate 4.42 we obtain

$$
|I| \leq\left\|\left|u-u_{h}^{L}\right|\right\|\left|\left\|\phi-\pi_{h}^{L} \phi \mid\right\| \lesssim h^{2}\|\psi\|_{L^{2}(\Omega) \times L^{2}(\Gamma)}\right.
$$

Term II. Using Lemma 4.5 we immediately get

$$
|I I| \lesssim h^{2}\|\psi\|_{L^{2}(\Omega) \times L^{2}(\Gamma)}
$$


Term III. Using Lemma 4.4 we obtain

$$
\begin{aligned}
\left|a\left(u_{h}^{L}, \pi_{h}^{L} \phi\right)-a_{h}\left(u_{h}^{L}, \pi_{h}^{L} \phi\right)\right| \lesssim & h^{2}\left\|\nabla_{\Gamma} u_{S, h}^{L}\right\|_{L^{2}(\Gamma)}\left\|\nabla_{\Gamma} \pi_{S, h}^{L} \phi_{S}\right\|_{L^{2}(\Gamma)} \\
& +h^{2}\left\|b \cdot u_{h}^{L}\right\|_{L^{2}(\Gamma)}\left\|b \cdot \pi_{h}^{L} \phi\right\|_{L^{2}(\Gamma)} \\
& +h\left\|\nabla u_{B, h}^{L}\right\|_{L^{2}\left(\mathcal{U}_{\delta}(\Gamma) \cap \Omega\right)}\left\|\nabla \pi_{B, h}^{L} \phi_{B}\right\|_{L^{2}\left(\mathcal{U}_{\delta}(\Gamma) \cap \Omega\right)}
\end{aligned}
$$

for $h \lesssim \delta \leq \delta_{0}$ and $h$ small enough. To show that the third term is actually of second order we shall use the Poincaré inequality

$$
\|v\|_{L^{2}\left(\mathcal{U}_{\delta}(\Gamma) \cap \Omega\right)} \lesssim\left(\delta / \delta_{0}\right)^{1 / 2}\|v\|_{H^{1}\left(\mathcal{U}_{\delta_{0}}(\Gamma) \cap \Omega\right)}, \quad 0<\delta \leq \delta_{0}
$$

See [7] for a proof of this inequality. We proceed in the following way

$$
\begin{aligned}
\left\|\nabla \pi_{B, h}^{L} \phi_{B}\right\|_{L^{2}\left(\mathcal{U}_{\delta}(\Gamma) \cap \Omega\right)} & \leq\left\|\nabla\left(\pi_{B, h}^{L} \phi_{B}-\phi_{B}\right)\right\|_{L^{2}\left(\mathcal{U}_{\delta}(\Gamma) \cap \Omega\right)}+\left\|\nabla \phi_{B}\right\|_{L^{2}\left(\mathcal{U}_{\delta}(\Gamma)\right)} \\
& \lesssim\left(h+\delta^{1 / 2}\right)\left\|\phi_{B}\right\|_{H^{2}\left(\mathcal{U}_{\delta_{0}}(\Gamma) \cap \Omega\right)} \\
& \lesssim\left(h+h^{1 / 2}\right)\left\|\phi_{B}\right\|_{H^{2}(\Omega)}
\end{aligned}
$$

where we used the fact that $\delta$ can actually be chosen such that $\delta \sim h$, see Lemma 4.4, and the interpolation error estimate 4.42 . The term $\left\|\nabla u_{B, h}^{L}\right\|_{L^{2}\left(\mathcal{U}_{\delta}(\Gamma)\right)}$ can be estimated using the same technique but we employ the energy norm error estimate 4.85 instead

$$
\begin{aligned}
\left\|\nabla u_{B, h}^{L}\right\|_{L^{2}\left(\mathcal{U}_{\delta}(\Gamma) \cap \Omega\right)} & \lesssim\left\|\nabla\left(u_{B, h}^{L}-u_{B}\right)\right\|_{L^{2}\left(\mathcal{U}_{\delta}(\Gamma) \cap \Omega\right)}+\left\|\nabla u_{B}\right\|_{L^{2}\left(\mathcal{U}_{\delta}(\Gamma) \cap \Omega\right)} \\
& \lesssim\left\|u-u_{h}\right\|+\delta^{1 / 2}\left\|\nabla u_{B}\right\|_{L^{2}\left(\mathcal{U}_{\delta}(\Gamma) \cap \Omega\right)} \\
& \lesssim\left(h+h^{1 / 2}\|u\|_{H^{2}(\Omega) \times H^{2}(\Gamma)}\right.
\end{aligned}
$$

Combining 4.96) and 4.97) we obtain

$$
I I I \lesssim\left(h^{2}+h\left(h+h^{1 / 2}\right)^{2}\right)\|\psi\|_{L^{2}(\Omega) \times L^{2}(\Gamma)} \lesssim h^{2}\|\psi\|_{L^{2}(\Omega) \times L^{2}(\Gamma)}
$$

Term $\boldsymbol{I} \boldsymbol{V}$. Using the fact that the jump term is consistent we obtain

$$
|I V|=\left|j_{h}\left(u-u_{h}, \phi-\pi_{h} \phi\right)\right| \leq||\left|u-u_{h}\right|\left\|_{\mathcal{F}}|| \phi-\pi_{h} \phi \mid\right\|_{\mathcal{F}} \lesssim h^{2}\|\psi\|_{L^{2}(\Omega) \times L^{2}(\Gamma)}
$$

where we used the energy estimate in Theorem 4.1 and the interpolation estimate in Lemma 4.1 .

We conclude the proof by collecting the estimates of Terms $I-I V$ and taking the supremum over all $\psi$ such that $\|\psi\|_{L^{2}(\Omega) \times L^{2}(\Gamma)}=1$.

\section{Estimate of the Condition Number}

Due to the different dimensions of the two coupled differential equations at the surface we shall see that it is natural to precondition the system in such a way that we seek $\left(v_{B, h}, v_{S, h}\right)$ such that the solution $\left(u_{B, h}, u_{S, h}\right)$ of (3.7) is given by

$$
\left(u_{B, h}, u_{S, h}\right)=\left(v_{B, h}, h^{1 / 2} v_{S, h}\right)
$$


The corresponding variational problem for $v_{h}=\left(v_{B, h}, v_{S, h}\right)$ takes the form: find $v=$ $\left(v_{B}, v_{S}\right) \in W_{h}$ such that

$$
\tilde{A}_{h}(v, w)=\tilde{L}_{h}(w) \quad \forall w \in W_{h}
$$

where the bilinear forms are defined by

$$
\tilde{A}_{h}(v, w)=A_{h}\left(\left(v_{B}, h^{1 / 2} v_{S}\right),\left(w_{B}, h^{1 / 2} w_{S}\right)\right), \quad \tilde{L}_{h}(w)=L_{h}\left(\left(w_{B}, h^{1 / 2} w_{S}\right)\right)
$$

We shall now estimate the condition number of the stiffness matrix $\tilde{A}$ associated with the bilinear form $\tilde{A}_{h}(\cdot, \cdot)$. Let $\left\{\varphi_{B, i}\right\}_{i=1}^{N_{B}}$ and $\left\{\varphi_{S, i}\right\}_{i=1}^{N_{S}}$ be the standard piecewise linear basis functions in $V_{B, h}$ and $V_{S, h} \oplus\left\langle 1_{\Gamma_{h}}\right\rangle$, respectively. Note that we have added the one dimensional space $\left\langle 1_{\Gamma_{h}}\right\rangle$ of constant functions on $\Gamma_{h}$. Define the following basis in the product space $V_{B, h} \times V_{S, h} \oplus\left\langle 1_{S, h}\right\rangle$ :

$$
\varphi_{i}= \begin{cases}\left(\varphi_{B, i}, 0\right) & 1 \leq i \leq N_{B} \\ \left(0, \varphi_{S, i-N_{B}}\right) & 1+N_{B} \leq i \leq N=N_{B}+N_{S}\end{cases}
$$

The expansion $v=\sum_{i=1}^{N} \widehat{v}_{i} \varphi_{i}$ defines an isomorphism

$$
\begin{aligned}
V_{B, h} \times V_{S, h} /\left\langle 1_{\Gamma_{h}}\right\rangle \oplus\left\langle 1_{S, h}\right\rangle & \rightarrow \mathbb{R}^{N_{B}} \times \mathbb{R}^{N_{S}} /\left\langle 1_{\mathbb{R}^{N_{S}}}\right\rangle \oplus\left\langle 1_{\mathbb{R}^{N_{S}}}\right\rangle \\
\left(v_{B}, v_{S} \oplus \bar{v}_{S} 1_{S, h}\right) & \mapsto\left(\widehat{v}_{B}, \widehat{v}_{S} \oplus \bar{v}_{S} 1_{\mathbb{R}^{N_{S}}}\right)
\end{aligned}
$$

where $v_{S}$ is the unique element in the equivalence classes of $V_{S, h} /\left\langle 1_{\Gamma_{h}}\right\rangle$ with $\int_{\Gamma_{h}} v_{S}=0$ and $\bar{v}_{S}=\left|\Gamma_{h}\right|^{-1} \int_{\Gamma_{h}} v_{S}$ is the meanvalue of $v_{S}$. If we introduce the mesh dependent $L^{2}$-norm

$$
\|v\|_{h}^{2}=\left\|v_{B}\right\|_{L^{2}\left(\mathcal{N}_{B, h}\right)}^{2}+\left\|v_{S}\right\|_{L^{2}\left(\mathcal{N}_{S, h}\right)}^{2}
$$

where the sets $\mathcal{N}_{B, h}$ and $\mathcal{N}_{S, h}$ are defined in (3.5), we have the following standard estimate

$$
c h^{-d}\|v\|_{h}^{2} \lesssim|\widehat{v}|_{N}^{2} \lesssim C h^{-d}\|v\|_{h}^{2}
$$

Let $\tilde{A}$ be the stiffness matrix with elements $a_{i j}=\tilde{A}_{h}\left(\varphi_{i}, \varphi_{j}\right)+J_{h}\left(\varphi_{i}, \varphi_{j}\right)$. The stiffness matrix is symmetric and has a one dimensional kernel consisting of a constant functions $v=\left(v_{B}, v_{S}\right)$, that satisfy $b \cdot v=b_{B} v_{B}-b_{S} v_{S}=0$. We shall estimate the condition number of $\tilde{A}$ as an operator on the invariant space $V=\mathbb{R}^{N_{B}} \times \mathbb{R}^{N_{S}} /\left\langle 1_{\mathbb{R}^{N_{S}}}\right\rangle$ defined by

$$
\kappa(\tilde{A})=|\tilde{A}|_{V}\left|\tilde{A}^{-1}\right|_{V}
$$

where $|x|_{N}^{2}=\sum_{i=1}^{N} x_{i}^{2}$ for $x \in \mathbb{R}^{N}$ and $|\tilde{A}|_{V}=\sup _{X \in V \backslash\{0\}} \frac{|\tilde{A} x|_{N}}{|x|_{N}}$ for $\tilde{A} \in \mathbb{R}^{N \times N}$. Next we introduce the discrete energy norm

$$
\|\| v \|_{h}^{2}=A_{h}(v, v)=a_{h}(v, v)+j_{h}(v, v)
$$

The proof of the estimate of the condition number follow the approach presented in $[8]$ and rely on a Poincaré and an inverse inequality which we prove next. 
Lemma 5.1 (Poincaré inequality) Independently of the mesh/boundary intersection it holds that

$$
\left\|\left(v_{B}, v_{S}\right)\right\|_{h} \lesssim\left\|\mid\left(v_{B}, h^{1 / 2} v_{S}\right)\right\|_{h} \quad \forall\left(v_{B}, v_{S}\right) \in W_{h}
$$

Proof. Using Lemma 3.3 in [5] and then adding and subtracting suitable terms and using the triangle inequality followed by a Poincaré inequality we obtain

$$
\begin{aligned}
\left\|v_{S}\right\|_{L^{2}\left(\mathcal{N}_{S, h}\right)}^{2} & \lesssim h\left\|v_{S}\right\|_{L^{2}\left(\Gamma_{h}\right)}^{2}+h j_{S}\left(v_{S}, v_{S}\right) \\
& \lesssim h\left\|\nabla_{\Gamma_{h}} v_{S}\right\|_{L^{2}\left(\Gamma_{h}\right)}^{2}+j_{S}\left(h^{1 / 2} v_{S}, h^{1 / 2} v_{S}\right) \\
& \lesssim\|\|\left(v_{B}, h^{1 / 2} v_{S}\right) \|_{h}^{2}
\end{aligned}
$$

Note that the Poincaré inequality is applicable on $\Gamma_{h}$ since $\int_{\Gamma_{h}} v_{S}=0$.

Next using the control provided by the jump term $J_{B}(\cdot, \cdot)$ followed by a Poincaré inequality we obtain

$$
\begin{aligned}
&\left\|v_{B}\right\|_{L^{2}\left(\mathcal{N}_{B, h}\right)}^{2} \lesssim\left\|v_{B}\right\|_{L^{2}\left(\Omega_{h}\right)}^{2}+h^{3} j_{B}\left(v_{B}, v_{B}\right) \\
& \lesssim\left\|P_{0} v_{B}\right\|_{L^{2}\left(\Omega_{h}\right)}^{2}+\left\|\nabla v_{B}\right\|_{L^{2}\left(\Omega_{h}\right)}^{2}+h^{3} J_{B}\left(v_{B}, v_{B}\right) \\
& \lesssim\left\|P_{0} v_{B}\right\|_{L^{2}\left(\Gamma_{h}\right)}^{2}+\left\|\left(v_{B}, h^{1 / 2} v_{S}\right)\right\|_{h}^{2} \\
& \lesssim\left\|\left(I-P_{0}\right) v_{B}\right\|_{L^{2}\left(\Gamma_{h}\right)}^{2}+\left\|v_{B}\right\|_{L^{2}\left(\Gamma_{h}\right)}^{2}+\left\|\left(v_{B}, h^{1 / 2} v_{S}\right)\right\|_{h}^{2} \\
& \lesssim\left\|\nabla v_{B}\right\|_{L^{2}\left(\Omega_{h}\right)}^{2}+b_{B}^{-2}\left\|b_{B} v_{B}-b_{S} h^{1 / 2} v_{S}\right\|_{L^{2}\left(\Gamma_{h}\right)}^{2} \\
&+b_{B}^{-2} b_{S}^{2}\left\|h^{1 / 2} v_{S}\right\|_{L^{2}\left(\Gamma_{h}\right)}^{2}+\left\|\left(v_{B}, h^{1 / 2} v_{S}\right)\right\| \|_{h}^{2} \\
& \lesssim b_{B}^{-2}\left\|h^{1 / 2} v_{S}\right\|_{L^{2}\left(\Gamma_{h}\right)}^{2}+\left\|\left(v_{B}, h^{1 / 2} v_{S}\right)\right\| \|_{h}^{2}
\end{aligned}
$$

Here $P_{0} v_{B}$ is the $L^{2}$-projection of $v_{B}$ onto constant functions on $\Omega_{h}$ and we added and subtracted suitable functions to control $P_{0} v_{B}$ using the coupling term together with the control of $\left\|h^{1 / 2} v_{S}\right\|_{\Gamma_{h}}^{2}$ provided by (5.12) and the fact that the constant $b_{B}>0$. Furthermore, the first inequality in (5.13) is a consequence of the inverse inequality

$$
\|v\|_{L^{2}\left(K_{1}\right)}^{2} \lesssim\|v\|_{L^{2}\left(K_{2}\right)}^{2}+h^{3}\left\|\left[n_{F} \cdot \nabla v\right]\right\|_{L^{2}(F)}^{2} \quad \forall v \in V_{B, h}
$$

that holds for each pair of elements $K_{1}$ and $K_{2}$ that share a face $F$. Iterating the inequality (5.14) we may control the elements at the boundary in terms of the elements in the interior of $\Omega_{h}$ as follows

$$
\|v\|_{L^{2}\left(K_{1}\right)}^{2} \lesssim\|v\|_{L^{2}\left(K_{N}\right)}^{2}+\sum_{i=1}^{N-1} h^{3}\left\|\left[n_{F} \cdot \nabla v\right]\right\|_{L^{2}\left(F_{i}\right)}^{2} \quad \forall v \in V_{B, h}
$$

see [11 for further details. Note that for sufficiently small mesh size the length $N$ of the shortest chain of elements that share an edge between an element that intersects the boundary and an interior element is uniformly bounded.

Combining the two estimates $(5.12)$ and $(5.13)$ the lemma follows directly. 
Lemma 5.2 (Inverse inequality) Independently of the mesh/boundary intersection it holds that

$$
\left\|\left(v_{B}, h^{1 / 2} v_{S}\right)\right\|_{h}^{2} \lesssim h^{-2}\left\|\left(v_{B}, v_{S}\right)\right\|_{h}^{2} \quad \forall\left(v_{B}, v_{S}\right) \in W_{h}
$$

Proof. Using standard estimates we obtain the following three estimates

$$
\begin{gathered}
b_{B} k_{B}\left\|\nabla v_{B}\right\|_{L^{2}\left(\Omega_{h}\right)}^{2}+\tau_{B} h^{3} j_{B}\left(v_{B}, v_{B}\right) \lesssim h^{-2}\left\|v_{B}\right\|_{L^{2}\left(\mathcal{N}_{B, h}\right)}^{2} \lesssim h^{-2}\left\|\left(v_{B}, v_{S}\right)\right\|_{h}^{2} \\
\left\|b_{B} v_{B}-b_{S} h^{1 / 2} v_{S}\right\|_{L^{2}\left(\Gamma_{h}\right)}^{2} \lesssim h^{-1} b_{B}\left\|v_{B}\right\|_{L^{2}\left(\mathcal{N}_{S, h}\right)}^{2}+b_{S}\left\|v_{S}\right\|_{L^{2}\left(\mathcal{N}_{S, h}\right)}^{2} \lesssim h^{-2}\left\|\left(v_{B}, v_{S}\right)\right\|_{h}^{2} \\
\left.b_{S} k_{S} h\left\|\nabla_{\Gamma_{h}} v_{S}\right\|_{L^{2}\left(\Gamma_{h}\right)}^{2}+\tau_{S} h j_{S}\left(v_{S}, v_{S}\right)\right) \lesssim\left(b_{S} k_{S}+\tau_{S}\right)\left\|\nabla v_{S}\right\|_{L^{2}\left(\mathcal{N}_{S, h}\right)}^{2} \lesssim h^{-2}\left\|v_{S}\right\|_{L^{2}\left(\mathcal{N}_{S, h}\right)}^{2}
\end{gathered}
$$

and thus the proof is complete.

Finally, we are ready to prove our final estimate of the condition number.

Theorem 5.1 The following estimate of the condition number of the stiffness matrix holds independently of the mesh/boundary intersection

$$
\kappa(\tilde{A}) \lesssim h^{-2}
$$

Proof. We need to estimate $|\tilde{A}|_{V}$ and $\left|\tilde{A}^{-1}\right|_{V}$. Starting with $|\tilde{A}|_{V}$ we have

$$
\begin{aligned}
|\tilde{A} \widehat{v}|_{V} & =\sup _{\widehat{w} \in \mathbb{R}^{N}} \frac{(\widehat{w}, \tilde{A} \widehat{v})_{N}}{|\widehat{w}|_{N}} \\
& =\sup _{w \in W_{h}} \frac{A_{h}\left(\left(v_{B}, h^{1 / 2} v_{S}\right),\left(w_{B}, h^{1 / 2} w_{S}\right)\right)}{\left|\left\|\left(w_{B}, h^{1 / 2} w_{S}\right) \mid\right\|_{h}\right.} \frac{\left\|\left(w_{B}, h^{1 / 2} w_{S}\right)\right\| \|_{h}}{\left\|\left(w_{B}, w_{S}\right)\right\|_{h}} \frac{\left\|\left(w_{B}, w_{S}\right)\right\|_{h}}{|\widehat{w}|_{N}} \\
& \lesssim h^{(d-2) / 2}\left\|\mid\left(v_{B}, h^{1 / 2} v_{S}\right)\right\| \|_{h} \\
& \lesssim h^{d-2}|\widehat{v}|_{N}
\end{aligned}
$$

where at last we used the estimate

$$
\left\|\left|\left(v_{B}, h^{1 / 2} v_{S}\right)\right|\right\|_{h} \lesssim h^{-1}\left\|\left(v_{B}, v_{S}\right)\right\|_{h} \lesssim h^{(d-2) / 2}|\widehat{v}|_{N}
$$

together with (5.16) and (5.8). Thus

$$
|\tilde{A}|_{V} \lesssim h^{d-2}
$$

Next we turn to the estimate of $\left|\tilde{A}^{-1}\right|_{V}$. Using (5.8) and (5.11), we get

$$
\begin{aligned}
|\widehat{v}|_{N}^{2} & \left.\lesssim h^{-d}||\left|\left(v_{B}, h^{1 / 2} v_{S}\right)\right|\right|_{h} ^{2} \lesssim h^{-d} A_{h}\left(\left(v_{B}, h^{1 / 2} v_{S}\right),\left(v_{B}, h^{1 / 2} v_{S}\right)\right) \\
& \lesssim h^{-d}(\widehat{v}, \tilde{A} \widehat{v})_{N} \lesssim h^{-d}|\widehat{v}|_{N}|\tilde{A} \widehat{v}|_{N}
\end{aligned}
$$

and thus we conclude that $|\widehat{v}|_{N} \leq C h^{-d}|\tilde{A} \widehat{v}|_{N}$. Setting $\widehat{v}=\tilde{A}^{-1} \widehat{w}$ we obtain

$$
\left|\tilde{A}^{-1}\right|_{N} \lesssim h^{-d}
$$

Combining estimates 5.22 and 5.24 of $|\tilde{A}|_{N}$ and $\left|\tilde{A}^{-1}\right|_{N}$ the theorem follows. 


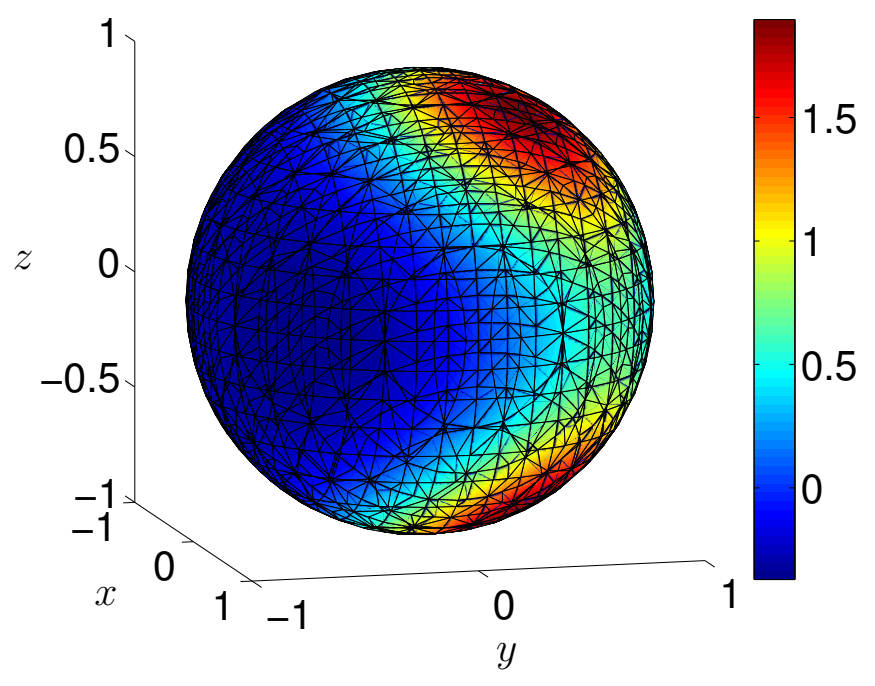

Figure 2: The solution $u_{S, h}$ with $h=0.13125$.

\section{$6 \quad$ Numerical results}

We consider an example where the domain $\Omega$ is the unit sphere, $k_{B}=k_{S}=1, b_{B}=b_{S}=1$, and $f_{B}$ and $f_{S}$ are choosen such that the exact solution is as in [7] given by

$$
\begin{aligned}
& u_{B}=e^{(-x(x-1)-y(y-1))} \\
& u_{S}=(1+x(1-2 x)+y(1-2 y)) e^{(-x(x-1)-y(y-1))}
\end{aligned}
$$

We study the convergence rate of the numerical solution $u_{h}=\left(u_{B, h}, u_{S, h}\right)$ and the condition number of the system matrix using the proposed finite element method. A direct solver is used to solve the linear systems. The stabilization parameters $\tau_{B}=\tau_{S}=10^{-2}$. We use a structured mesh for $\Omega_{0}$ and the mesh parameter $h=h_{x}=h_{y}=h_{z}$.

To represent the boundary $\Gamma$ we use the standard level set method. We define a piecewise linear approximation to the distance function on $\mathcal{K}_{0, h}$ and $\Gamma$ is approximated as the zero level set of this approximate distance function. Thus, $\Gamma_{h}$ is represented by linear segments on $\mathcal{K}_{0, h}$. The normal vectors are computed from the linear segments.

The solution $u_{S, h}$ with $h=0.13125$ and the triangulation of $\Gamma_{h}$ are shown in Fig. 2. The convergence of $u_{h}$ in both the $L^{2}$ norm and the $H^{1}$ norm are shown in Fig. 3 . We have as expected first order convergence in the $H^{1}$ norm and second order convergence in the $L^{2}$ norm. The spectral condition number of the matrix $\tilde{A}$ associated with the bilinear form $\tilde{A}_{h}(\cdot, \cdot)$ (see equation (5.3)) is shown for different mesh sizes in Fig. 4 

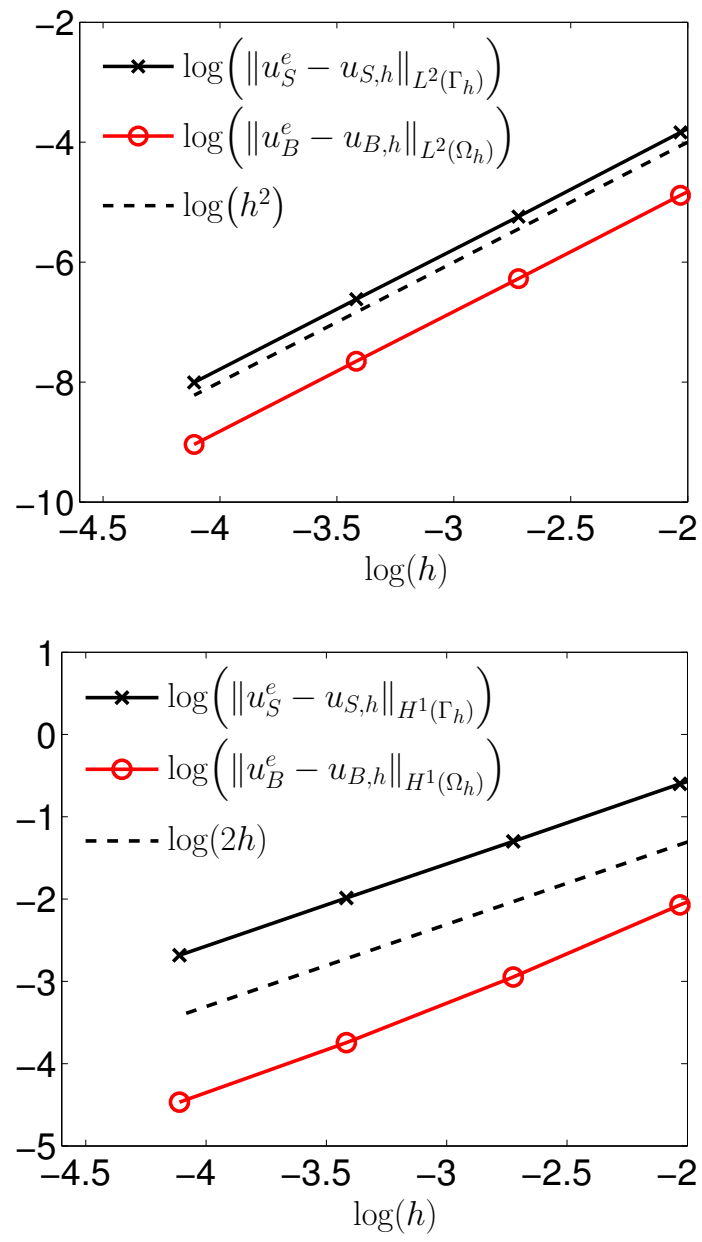

Figure 3: Convergence of $u_{B}$ and $u_{S}$. Upper panel: The error measured in the $L^{2}$ norm versus mesh size. Lower panel: The error measured in the $H^{1}$ norm versus mesh size. 


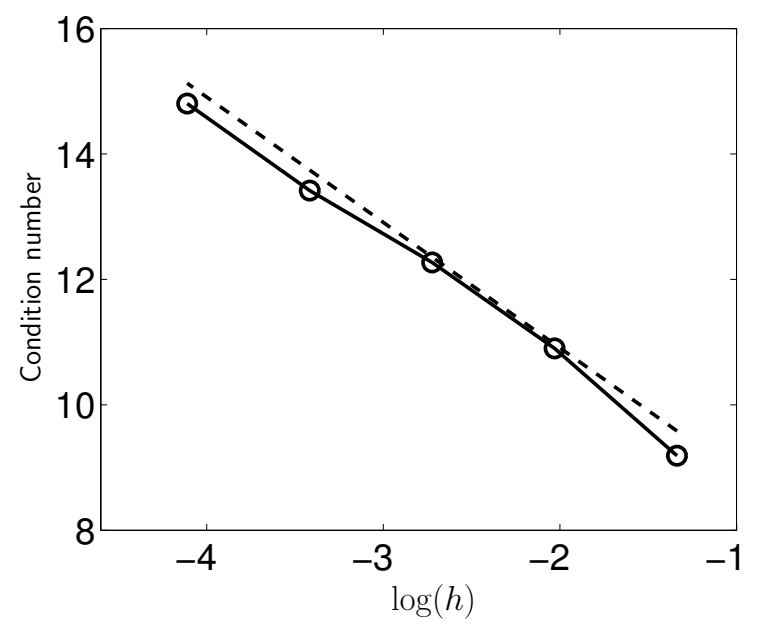

Figure 4: The spectral condition number of the matrix $\tilde{A}$ versus mesh size. The dashed line is proportional to $h^{-2}$.

\section{Appendix}

Here we will give some details on the inequalities (4.5). First we recall that

$$
q_{h}(x)=x+\gamma_{h}(x) n(x) \quad x \in \Gamma
$$

Now using the defintion of the closest point mapping

$$
y=p(y)+\rho(y) n^{e}(y) \quad y \in \Gamma_{h}
$$

Setting $x=p(y)$ in 6.2 we have

$$
y=p(y)+\gamma_{h}(p(y)) n^{e}(y) \quad y \in \Gamma_{h}
$$

and therefore, by uniqueness, $\rho(y)=\gamma_{h}(p(y)), \forall y \in \Gamma_{h}$. Thus we have $\gamma_{h}=\rho^{L}$ and we immediately obtain the first inequality in (4.5) since

$$
\left\|\gamma_{h}\right\|_{L^{\infty}(\Gamma)}=\left\|\rho^{L}\right\|_{L^{\infty}(\Gamma)}=\|\rho\|_{L^{\infty}\left(\Gamma_{h}\right)} \lesssim h^{2}
$$

Next using 4.31) we have the identity

$$
\nabla_{\Gamma} \gamma_{h}=\nabla_{\Gamma} \rho^{L}=D F_{h, \Gamma}^{T}\left(\nabla_{\Gamma_{h}} \rho\right)^{L}=D F_{h, \Gamma}^{T}\left(P_{\Gamma_{h}} n^{e}\right)^{L}
$$

Estimating the right hand side using (4.18) and 4.70 we finally obtain

$$
\left\|\nabla_{\Gamma} \gamma_{h}\right\|_{\Gamma} \lesssim\left\|\nabla_{\Gamma_{h}} \rho\right\|_{\Gamma_{h}} \lesssim\left\|P_{\Gamma_{h}} n^{e}\right\|_{\Gamma_{h}} \lesssim h
$$

which is the second bound in 4.5. 


\section{References}

[1] M. R. Booty and M. Siegel, A hybrid numerical method for interfacial fluid flow with soluble surfactant, J. Comput. Phys. 229 (2010) 3864-3883.

[2] S. C. Brenner and L. R. Scott, The mathematical theory of finite element methods, Springer Verlag, 3rd ed. (2008).

[3] E. Burman; S. Claus, P. Hansbo, M. G. Larson, A. Massing, CutFEM: discretizing geometry and partial differential equations, Int. J. Num. Meth. Engrg. Submitted. 2014.

[4] E. Burman; P. Hansbo, Fictitious domain finite element methods using cut elements: II. A stabilized Nitsche method, Appl. Numer. Math. 62 (4) (2012) 328-341.

[5] E. Burman, P. Hansbo, M. G. Larson, A stable cut finite element method for partial differential equations on surfaces: the Laplace-Beltrami operator, arXiv:1312.1097 (2013).

[6] G. Dziuk and C. M. Elliott, Finite element methods for surface PDEs, Acta Numerica, $22,(2013)$, pp $289-396$

[7] C. M. Elliott and T. Ranner, Finite element analysis for a coupled bulk-surface partial differential equation, IMA J. Numer. Anal. 33 (2) (2013) 377-402.

[8] A. Ern, J. L. Guermond, Evaluation of the condition number in linear systems arising in finite element approximations, ESAIM: Mathematical Modelling and Numerical Analysis 40 (01), 29-48.

[9] Y. Georgievskii, E. S. Medvedev, and A. A. Stuchebrukhov, Proton transport via the membrane surface, Biophys J. 82(6) (2002) 2833-2846.

[10] P. Hansbo, M. G. Larson, and S. Zahedi, A cut finite element method for a Stokes interface problem, arXiv:1205.5684, (2012).

[11] A. Massing, M. G. Larson, A. Logg and M. E. Rognes, A stabilized Nitsche fictitious domain method for the Stokes problem, arXiv:1206.1933, accepted for publication in Journal of Scientic Computing, (2013).

[12] M. A. Olshanskii, A. Reusken, J. Grande, A finite element method for elliptic equations on surfaces, SIAM J. Numer. Anal. 47 (2009) 3339 - 3358.

[13] M. A. Olshanskii, A. Reusken, A finite element method for surface PDEs: matrix properties, Numer. Math. 114 (3) (2010) 491-520 . 